\title{
Antiprotons and the Crystal Ball
}

\author{
CB-99-010
}

\author{
Chris Allgower ${ }^{1}$ and David Peaslee ${ }^{2}$ \\ ${ }^{1}$ High Energy Physics Division, Argonne National Laboratory, Argonne, IL 60439 \\ ${ }^{2}$ University of Maryland, Physics Dept., College Park, MD 20742
}

September 21, 1999

\section{Introduction}

During the 1998 running of the Crystal Ball experiment, a couple of brief test runs were done with the C6 beamline tuned to produce antiprotons. Specifically, one shift on July 29th produced runs 329-334 with the beam momentum set for $650 \mathrm{MeV} / \mathrm{c}$, run 355 was done on July 31st with a $550 \mathrm{MeV} / \mathrm{c}$ beam momentum, and runs 926-929 were obtained in a single shift on November 3rd, also with a beam momentum of $550 \mathrm{MeV} / \mathrm{c}$. The beam tune for the November data was greatly superior to that of the July data, however. Therefore, only the November data have been analyzed in detail, and the results of this analysis are presented in this note. Due to the paucity of statistics that were obtained, it was decided not to attempt to publish the results. However, the results are valuable as a tool for planning a possible future program of dedicated measurements of antiprotonproton annihilation into all-neutral final states using the Crystal Ball. The data in fact show that the Crystal Ball with its large angular acceptance and multi-photon capability would be an excellent detector for such an experiment. Only one other such experiment (the Crystal Barrel experiment at CERN/LEAR) has ever been done in the past.

Also in this document is a survey of published results for old antiproton-proton experiments, some discussion of physics issues that are relevant to measurements of $\bar{p}+p \rightarrow$ all-neutral final states, and some estimates about the kinds of experiments we could contemplate doing in the C6 and D6 lines at the AGS. 


\section{Data Analysis and Results}

The neutral analysis consisted of selecting good antiproton-proton events via two cuts on the raw data, and then analyzing them one by one with the aid of a separate FORTRAN program and a fair amount of human effort and judgment. A good event had two characterstics: first, the S1-S2 time of flight was consistent with being an antiproton in the incident beam and second, the total energy deposited in the ball had to be consistent with antiproton-proton annihilation into some number of photons where all of the photons were captured in the Crystal Ball, i.e., there was no missing mass or energy.

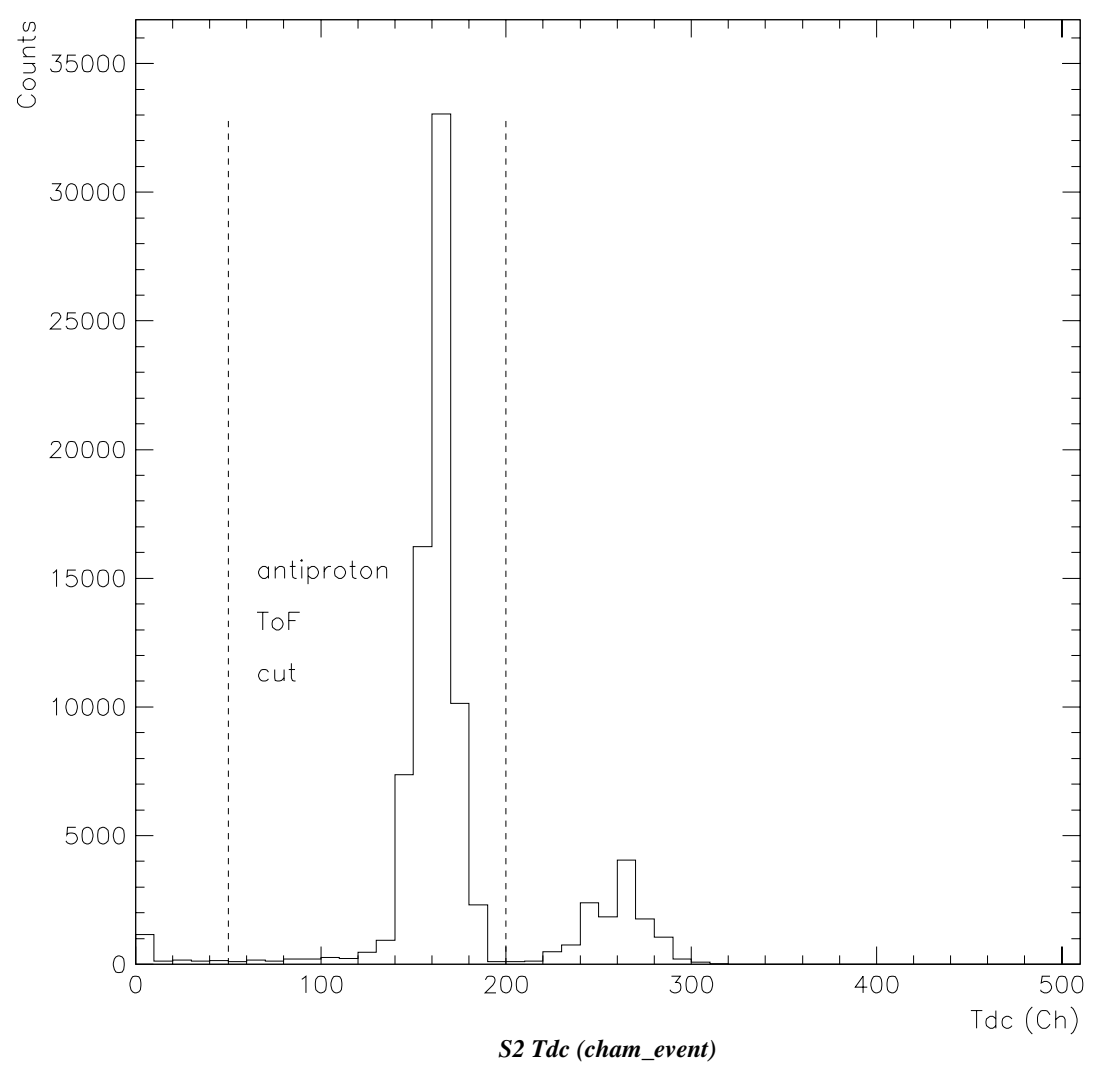

Figure 1: Time of Flight S1 to S2 with cuts indicated for selecting antiprotons. Events in the right-hand peak are pions. (Note that time is reversed in this spectrum. Early events appear at the large TDC channels.) 
Figure 1 shows the time of flight cut that selected incident antiprotons in the beam. Due to their large mass difference with pions and kaons, the antiprotons arrived with much lower velocities and were therefore easily separable from other particle species in the incident beam.
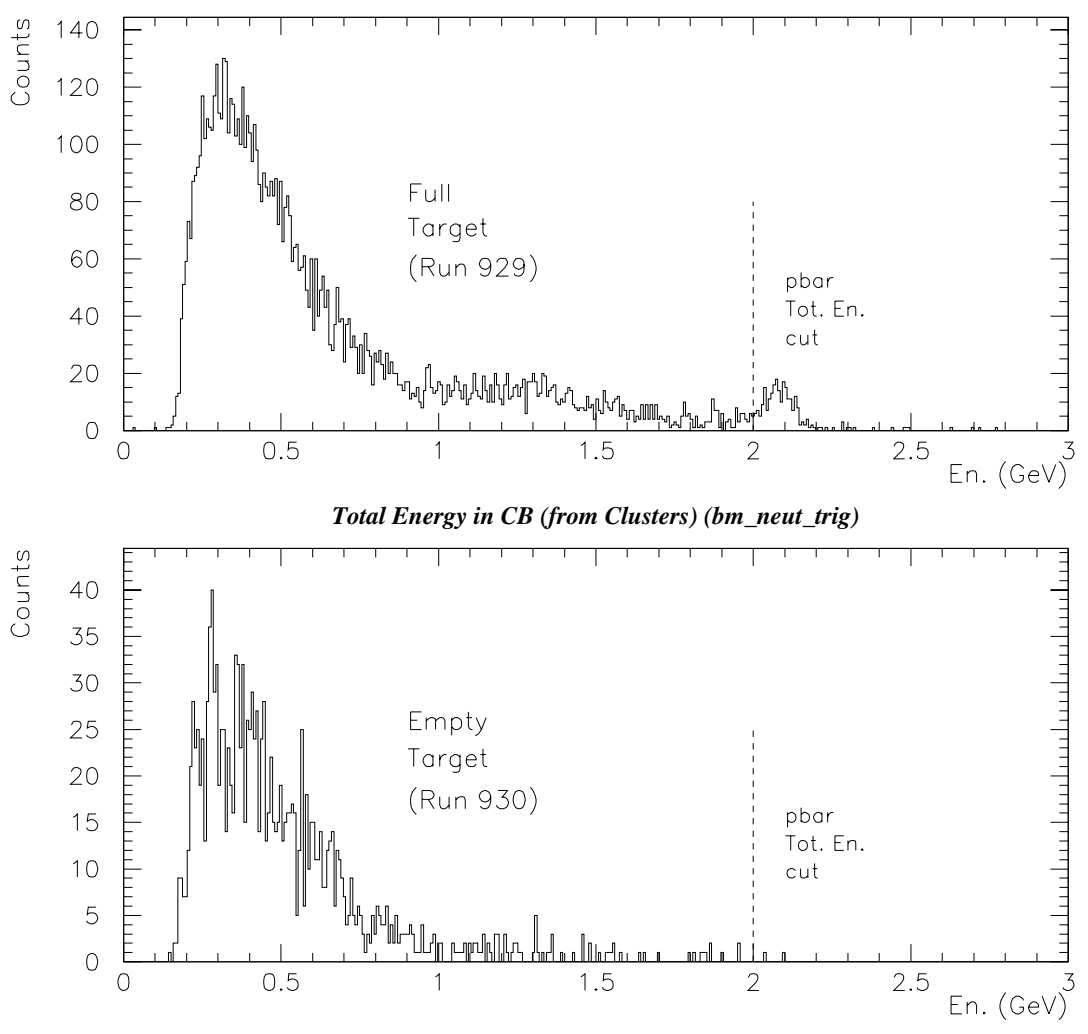

Total Energy in CB (from Clusters) (bm_neut_trig)

Figure 2: Total Energy Deposited in the Crystal Ball for events which passed the antiproton time of flight cut. Spectra for runs with the liquid hydrogen target full (top) and empty (bottom) are compared. Above the cut at $2.0 \mathrm{GeV}$ there was virtually no background from the target walls.

Figure 2 shows the total energy spectra (from the sum of all cluster energies) for events in the antiproton time of flight cut. A clear bump above $2.0 \mathrm{GeV}$ indicates the events for which all the final-state energy was captured. Only two "good" events 
Table 1: Statistics of Runs 927-930

\begin{tabular}{|r|r|r|r|r|l|}
\hline Run & Start & End & $\begin{array}{r}\text { Run } \\
\text { Length }\end{array}$ & $\begin{array}{r}\text { \# of Good } \\
\text { Events }\end{array}$ & \\
& Time & Time & 29 & Full Tgt., CB Thresh. $=3.0 \mathrm{~V}$ \\
927 & $17: 28$ & $17: 57$ & $29 \mathrm{~min}$. & 52 & Full Tgt., CB Thresh. $=3.0 \mathrm{~V}$ \\
928 & $17: 59$ & $18: 40$ & $41 \mathrm{~min}$. & 250 & Full Tgt., CB Thresh. $=1.0 \mathrm{~V}$ \\
929 & $18: 54$ & $22: 48$ & $230 \mathrm{~min}$. & 331 & Totals for Full Target \\
\hline \hline & & & $300 \mathrm{~min}$. & 2 & Empty Tgt., CB Thresh. $=3.0 \mathrm{~V}$ \\
\hline \hline 930 & $23: 04$ & $00: 00$ & $56 \mathrm{~min}$. & &
\end{tabular}

were observed in fifty six minutes of empty target running, indicating that the number of "good" events from the walls of the target container was only about $3 \%$ of the total when running with the target full. Table 1 Shows some statistics concerning runs 927-930, including the numbers of events which passed both cuts. Note that Run 929 contained most of the statistics. Runs 927 and 928 were run with higher thresholds on the ball, but in all cases the thresholds were set far below the region selected by the total energy cut. The observant reader will notice a discrepancy of 4 minutes in the run length of Run 929 in Table 1. Four minutes were subtracted due to a comment in the logbook to the effect that the beam was off for that length of time while Run 929 was in progress. The rate for good events was very slow, as is evident in the table. A total of only 331 good events were observed in 300 minutes of full-target running. It was therefore possible to attempt to analyze the good events one at a time by hand.

Two routines were written to do analysis of the photon clusters of the "good" events in the ball. The first, inv_miss_all, calculated collective kinematic quantities obtained by combining all possible combinations of the $n$ clusters in the event. The kinematic quantities were written to a text file for later analysis by the second routine, im_srch.f, which did preliminary searches on the text file to identify the combinations of clusters that were candidates for being pions, eta mesons, omegas, etaprimes, or phis. An attempt was then made to piece together the event by hand from the resulting list of candidates. 
Appendix A contains listings of the source codes of the include file inv_miss_all.inc, the fortran routine inv_miss_all, and the separate program im_srch.f. For an event with $n$ clusters, there are $2^{n}$ possible ways of picking $m \leq n$ clusters to calculate invariant masses and other kinematic quantities with. For each choice of $m$ clusters, the routine inv_miss_all calculated the invariant mass, the three momentum components, the scattering angle, the total energy, the missing mass, the missing momentum, the missing energy, and the "missing" particle's scattering angle. These quantities were written to an ascii file during the running of the analyzer. After running the analyzer, the ascii file was searched for particle candidates by the program im_]srch. In practice, the user used im_srch in tandem with the single event display (SED) from the analyser. For each "good" event, the user would first display it on the screen using the SED. From the SED one obtained the event number. Program im_srch would then be told to search the kinematics of that event. The rules im_srch used for identifying particle candidates are shown in Table 2.

Table 2: Search criteria used by im_srch.

\begin{tabular}{|c|c|c|l|l|}
\hline $\begin{array}{c}\text { Part. } \\
\text { Type }\end{array}$ & $\begin{array}{c}\text { Inv. Mass } \\
\text { Cut (Rel.) }\end{array}$ & $\begin{array}{c}\text { Inv. Mass } \\
\text { Cut (Abs.) }\end{array}$ & $\begin{array}{l}\text { Numbers of } \\
\text { Clusters }\end{array}$ & $\begin{array}{l}\text { Decay Channels } \\
\text { Selected for }\end{array}$ \\
\hline \hline$\pi^{\circ}$ & $m_{\pi^{\circ}} \pm 30 \mathrm{MeV}$ & $0.105-0.165 \mathrm{GeV} / c^{2}$ & 2 & $\pi^{\circ} \rightarrow 2 \gamma$ \\
$\eta$ & $m_{\eta} \pm 47 \mathrm{MeV}$ & $0.500-0.594 \mathrm{GeV} / c^{2}$ & 2,6 & $\eta \rightarrow 2 \gamma, 3 \pi^{\circ}$ \\
$\omega$ & $m_{\omega} \pm 69 \mathrm{MeV}$ & $0.713-0.851 \mathrm{GeV} / c^{2}$ & 3,7 & $\omega \rightarrow \pi^{\circ} \gamma, \eta \gamma$ \\
$\eta^{\prime}$ & $m_{\eta^{\prime}} \pm 88 \mathrm{MeV}$ & $0.870-1.046 \mathrm{GeV} / c^{2}$ & $2,4,6,8,10$ & $\eta^{\prime} \rightarrow 2 \gamma, \omega \gamma, 2 \pi^{\circ} \eta$ \\
$\phi$ & $m_{\phi} \pm 94 \mathrm{MeV}$ & $0.925-1.113 \mathrm{GeV} / c^{2}$ & 3,8 & $\phi \rightarrow \pi^{\circ} \gamma, \eta \gamma$ \\
\hline
\end{tabular}

The output of im_srch was a listing of those cluster numbers which when grouped together satisfied the criteria listed in Table 2. From that point forward, the user worked by hand. The adopted method was to first write down the cluster number pairs from the 2-cluster pion and eta combinations on a sheet of paper in separate columns. The six-cluster eta combinations were also investigated to see if any of them corresponded to three separate pairs of clusters in the pion list. If so, those six-cluster groups were added 
to the eta list. Then the list of three-cluster omega candidates was scanned and any omega triplets which also contained pion or eta pairs were written down under a column of omega candidates on the sheet. (This step usually trimmed down the list of omega candidates that im_srch had identified by a considerable amount.) The three-cluster phi candidates were treated in similar fashion to the 3-cluster omega options, and surviving candidates were listed under a phi column on the sheet. (The $K_{L} K_{S}$ decay mode of the phi meson was not considered because the $K_{L}$ would not have deposited energy in the ball in such a way as to satisfy the total energy cut.) If there were any valid six-cluster eta decays, and there were were seven-cluster omega options in the list from im_srch, these were checked to see if they might be $\omega \rightarrow \eta \gamma$ with the eta having decayed to three pions. Only after all the pion, eta, and omega options had been identified were the etaprime candidates investigated. Two-cluster etaprime candidates always made the list, four-cluster options made the list whenever three of the four clusters corresponded to at least one of the three-cluster omega candidates, six-cluster options made the list if the six clusters could be understood as having come from an eta decay and two pions, eightcluster options made the list if they consisted of a seven-cluster omega option plus an odd photon, and ten-cluster options had to be a six-cluster eta option (from the written sheet) plus two extra pion cluster-pairs (in other words, five pions, three of which could have come from an eta decay). At the end of this process one had a written list of cluster groupings that corresponded to particle candidates that were all consistent with the most common all-neutral decay modes of the particles in question. Depending on the complexity of the event, it could take the user anywhere from a minute or two to about forty minutes to reach this point for a given event.

Once the lists of candidate particles had been written down, the final phase of the analysis of the event consisted of trying to piece the candidates together in such a way as to account for all of the observed clusters. Any combinations that did so were written down in a list of reaction candidates provided they were below threshold for a 550 $\mathrm{MeV} / \mathrm{c}$ incident proton beam. Often one would obtain several valid reaction candidates, of which some number may have been permutations of clusters which coresponded to the same reaction channel. For example, four-cluster etaprime options could often be 
reconstructed in multiple ways from the same four clusters. A six-cluster etaprime eta event might therefore have two or three etaprime eta options in the final list of reaction candidates because of the multiple ways in which the etaprime might have decayed. In order to keep permutations straight, the order of the cluster numbers for the particle options was always written in a consistent way so that one could tell which clusters were to be interpreted as odd photons and which were coming from pion or eta decays. After attempting to peice together the jigsaw puzzle of each event, anywhere from zero to a few dozen entries would appear on the list of reaction candidates. (In the most extreme case, a ten-cluster event resulted in 87 different ways in which all ten clusters could be accounted for.)

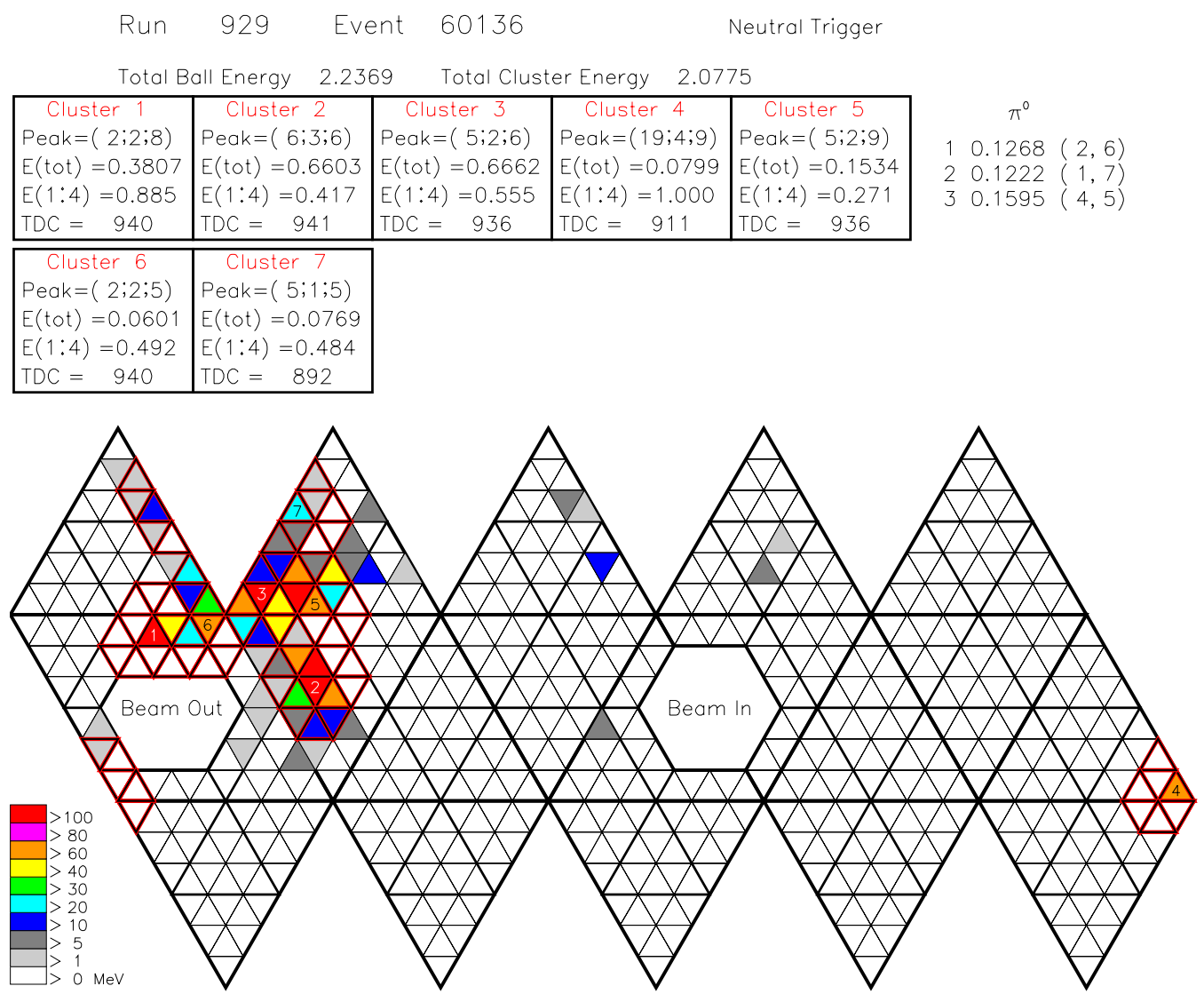

Figure 3: Unreconstructable event, probably from $\bar{p} p \rightarrow \bar{n} n$ where the antineutron annihilated in the sodium iodide crystals of the ball. 
There were several ways in which one could end up with no options being obtained. In about $5 \%$ of cases, one would have trouble reconstructing the event, and by looking at the single event display one would notice that the total energy in the ball seemed to be a little too high overall. Indeed, the total energy peak in Fig. 2 has a centroid at about $2.070 \mathrm{GeV}$, whereas from the known proton mass and an incident beam momentum of $550 \mathrm{MeV} / \mathrm{c}$ one would expect to see the peak centered at $2.026 \mathrm{GeV} / \mathrm{c}$. This suggests that the calibration of the ball may have been causing the analyzer to systematically overestimate the photon energies by about $2 \%$, thereby causing some difficulties for the event reconstruction. The calibration of the ball was done using pion charge exchange, which produced photons of much lower energy than those typically encountered in antiproton reactions. One might expect therefore that the calibration might have been less reliable at the higher photon energies as well. No attempt was made in the analysis to correct the calibration to rectify this feature. Another less common problem was that at times one encountered an event where two clusters were overlapping in the crystals. In such cases the clustering algorithm tended to lump shared crystals into the cluster that gave the higher energy, thereby obtaining wrong energies for both clusters. This problem was especially evident for the high-energy forward pions produced by the reaction $\bar{p} p \rightarrow 2 \pi^{\circ}$. (See Fig. 11 for an SED picture of such a $2 \pi^{\circ}$ event.) In such events the forward pion nearly always had an opening angle so small that its clusters overlapped. (However, $2 \pi^{\circ}$ events were easily identifiable because of their characteristic four clusters pair-wise nearly back to back in the ball.) Other types of events tended not to have overlapping clusters with great regularity. Occasionally, one would see an event where it was obvious from looking at the SED that it was going to be impossible to reconstruct the event. In such events all the energy in the ball was located on one side of the ball and one could see immediately that the overall transverse momentum in the ball was completely unbalanced. Many of these events had enormous superclusters of several contiguous clusters in a large patch that was lighting up a whole region of the ball. The likely cause of these were the reaction $\bar{p} p \rightarrow \bar{n} n$, where the outgoing antineutron had annihilated completely in the sodium iodide crystals. (See Fig. 3 for a view of such an event from the SED.) Overall, one failed to obtain any reconstruction of an event only about $10 \%$ of the time. 


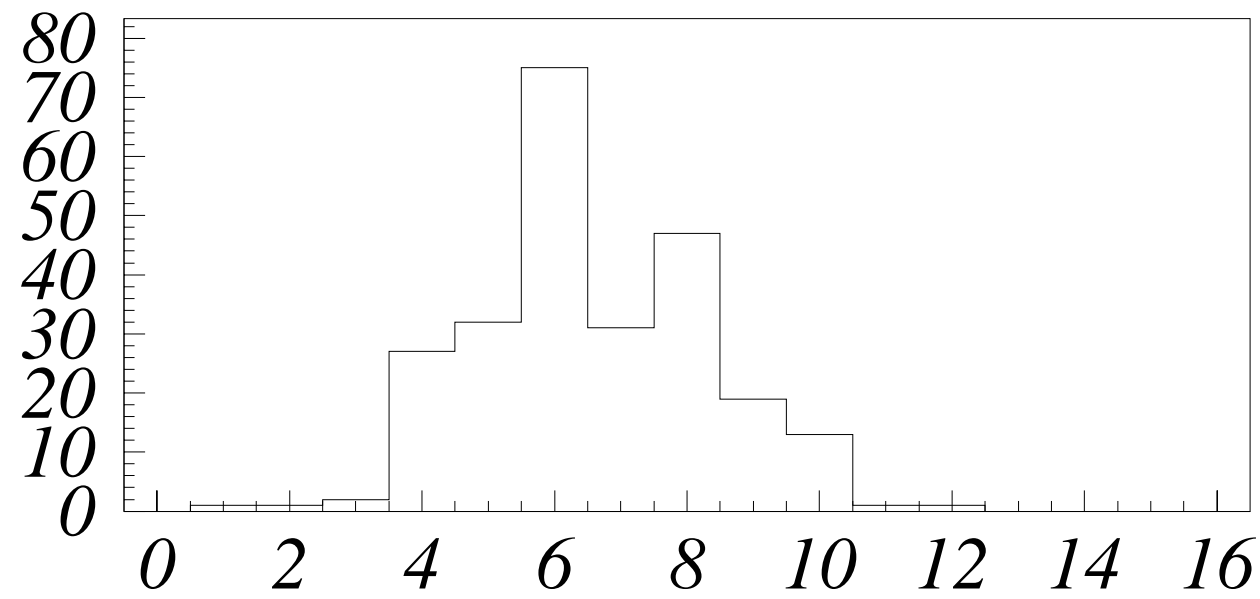

Figure 4: Number of clusters per event for "good" events.

In the $90 \%$ of cases where one was able to obtain at least one valid reaction option that used all the clusters, one often had to try to make judgments about the different options and apply tests to see if some options could be ruled out. For events which had relatively few clusters the reconstruction process tended to yield comparatively unambiguous results. However, some events had upwards of ten clusters and could therefore be troublesome to identify with confidence. The distribution of the number of clusters per event for the "good" events of run 929 is shown in Figure 4 to illustrate the level of complexity involved in some of the events that were observed. In particular, one sees immediately excesses in the six-cluster and eight-cluster events. The 6-cluster bin was dominated by $3 \pi^{\circ}$ events, and the eight-cluster bin had several strong channels contributing to it.

In fact, the eight-cluster events tended to be some of the most difficult to identify because of the many channels involved, as well as the fact that several channels had identical signatures at least at the rather crude level of the analysis performed here. For example, an event that had four $\pi^{\circ}$ s in it quite often could also have easily been $\eta^{\prime} \pi^{\circ} \pi^{\circ}$, where the $\eta^{\prime}$ decayed to $\omega \gamma$ with the omega decaying via $\gamma \pi^{\circ}$. Furthermore, $4 \pi^{\circ}$ events 
could have been produced via $2 K_{s}$ production in addition to nonstrange channels.

In general, events with ambiguous interpretations were of two types - those with two final-state particles (which subsequently decayed into photons) and those in which three or more particles had been directly produced in the final state at least in some sense. Examples of channels in the former category might be $\phi \eta^{\prime}$ or $\eta \pi^{\circ}$, and examples of the latter category included $3 \pi^{\circ}, \eta \pi^{\circ} \pi^{\circ}$, and $\eta^{\prime} \eta \pi^{\circ}$. In cases where there were reaction options for two-particle final states, it was possible to test the two-particle kinematics of the situation. For the two groupings of clusters that corresponded to the two finalstate particles, one could pick out the scattering angle and the expected angle of the other particle from the table of kinematic quantities that inv_miss_all had calculated. If both pairs of angles matched to within about $\pm 15^{\circ}$, the two-particle reaction option was considered to be a valid option. If the angles didn't match, the option was discarded from the list of reaction options. In this way, many of the 2-particle reaction options were discarded. The azimuthal angles of particles in two-particle final states were not checked for coplanarity. No similar tests were done for final state options in the three-ormore particle category. Although the attempt was made to match invariant and missing masses for groups of clusters in two-particle final state options, this test was given little weight because in practice the invariant mass and missing mass resolutions were too poor to allow this technique to work well. There were two reasons for this. In the first place one was dealing with high-energy photons, so the absolute energy resolution of the individual clusters was reduced, and in the second place one was attempting to calculate collective invariant and missing masses from groupings of upwards of six to eight clusters or more, which further added to the uncertainty. In particular, if one of the particles was a pion it was common to obtain an imaginary value for the missing mass off the clusters of the other particle because several high-energy photons were having to cancel out rather precisely to obtain a missing mass that was not far from zero on the overall energy scale. For this reason, angle matching was done, but the matching of invariant vs. missing masses was not given much trust, although it was always checked.

For eight-cluster events which had recontruction options for four $\pi^{\circ}$ s, a special check was performed to look for possible $2 K_{s}$ production. All pairs of pions were first 
checked to see if they had invariant masses that corresponded roughly to kaon masses. If there was a way of grouping two pairs of pions together so that both pion paris had kaon invariant masses, then those groupings of clusters were subjected to the same 2-particle angle checks as for other 2-particle options. If the angles matched, then the option was declared a possible $2 K_{s}$ candidate.

In general it was difficult to decide how to classify the $4 \pi^{\circ}$ events. In nearly all cases such events looked either like $2 K_{s}$ events or $\eta^{\prime} \pi^{\circ} \pi^{\circ}$ events. (There were only three exceptions - an event that appeared to be $\eta \pi^{\circ}$ with the eta having decayed to $3 \pi^{\circ}$ s, an event that was ultimately classified as $\eta^{\prime} \eta^{\prime}$, and a single direct $4 \pi^{\circ}$ event that did not look like it had come from any other channel.) Undoubtedly, many of the events in the $\eta^{\prime} \pi^{\circ} \pi^{\circ}$ and $2 K_{s}$ categories were probably simple $4 \pi^{\circ}$ events, but it was too difficult to tell them apart with any confidence. Clearly, eight-cluster channels will be difficult to unravel in the data analysis of a full-scale experiment of this kind. (Happily, one can be pretty sure that the $\eta^{\prime} \pi^{\circ} \pi^{\circ}$ channel was indeed observed, however, because it also showed up in a few six-cluster and ten-cluster events as well.)

Even after applying various tests to the two-particle reaction options, one was often left with multiple possible identifications of an event. At that point one was forced to hazard a guess based on one's own judgment. As one progressed through the data, statistics began to accumulate to indicate which channels tended to have the largest cross sections. Based on the strength of such experience, one could weight one's guess by such probabilities in making choices between options, but in many cases one could never make a unique identification for sure. Accordingly, three levels of "certainty" were adopted for event identifications. A "certain" event was one for which the identification of the event appeared to be unique with no other valid options present. An "uncertain" event was one for which either one had had to pick between two or more possible options, or one option had been found, but for whatever reason one was unsure of one's own identification. (For example, the pion invariant mass cuts used by im_srch may have been set slightly too narrow, so that it at times could miss cluster pairs with invariant masses slightly outside of the pion cut window. In such cases one would have difficulty reconstructing the event, but by looking at the SED one could identify likely cluster pairs that could have been 
missed as $\pi^{\circ}$ 's by im_srch. If the event could only be sensibly reconstructed by stretching things in this manner, it was put into the "uncertain" category.) Also see Fig. 15 for a specific example of an "uncertain" event. The third level of uncertainty was reserved for events which one was simply unable to make sense out of. Naturally there were some grey areas between the boundaries of the three certainty levels, and almost certainly mistakes were made in identifying events, as is to be expected when one is analyzing individual events by hand. In addition, splitoffs in photon showers in the sodium iodide crystals of the ball could also have accounted for some extra clusters. In addition, based on estimates done by Sasha Starostin of the frequency of splitoffs in other Crystal Ball data (splitoffs occur about $2.7 \%$ of the time), one could expect that about nine of the 333 total events might have had splitoffs in them.

Table 3: Table of events for which reconstruction was unsuccessful, successful. Numbers in upper half of table are the numbers of clusters in the individual events that could not be reconstructed.

\begin{tabular}{|c|c|c|c|c|c|}
\hline $\begin{array}{l}\text { Problem } \\
\text { w/Event }\end{array}$ & $\begin{array}{l}\text { Run } 927 \\
\text { Full Tgt }\end{array}$ & $\begin{array}{l}\text { Run } 928 \\
\text { Full Tgt }\end{array}$ & $\begin{array}{l}\text { Run } 929 \\
\text { Full Tgt }\end{array}$ & $\begin{array}{l}\text { Run } 930 \\
\text { MT Tgt }\end{array}$ & Totals \\
\hline $\bar{n} n ?$ & & & 467251 & & 6 \\
\hline & & & 76668610 & & \\
\hline Too High & 86 & 6 & 867461066 & & 18 \\
\hline Couldn't & & & & & \\
\hline Reconstruct & 76 & 889 & 969 & & 8 \\
\hline Total Bad & & & & & \\
\hline Events & 4 & 4 & 24 & 0 & 32 \\
\hline Tot. Renstd. & & & & & \\
\hline Events & 25 & 48 & 226 & 2 & 301 \\
\hline $\begin{array}{l}\text { Grand Total } \\
\text { of Events }\end{array}$ & 29 & 52 & 250 & 2 & 333 \\
\hline
\end{tabular}


Table 4 contains the full listing of reconstructed events that were obtained by the event reconstruction process. The reaction channels are listed in order of frequency and "certainty." Each run has a separate column listing the events in that run. Within the run columns, each reconstructed event is listed by the number of clusters in the event. Underlines distinguish events in the "uncertain" category from those in the "certain" category, and the numbers appear in the order in which the events they represent were found in each run. (In this way, one can easily see how often various channels appeared in events with different numbers of clusters.) Finally, the total numbers of events in the "uncertain" and "certain" categories are listed in the right-hand column together with the sums of the counts in both categories. Table 5 contains a specific breakdown of events which are included under various categories within Table 4, but which could have been alternatively classified as $4 \pi^{\circ}$ events. Note that the $K_{s} K_{s}$ and $\eta^{\prime} \pi^{\circ} \pi^{\circ}$ categories likely contain many indistiniguishable $4 \pi^{\circ}$ background events. Table 3 contains information about those additional events for which reconstruction was not successful, as well as overall totals of events for which reconstruction was or was not possible.

Table 4 shows the abundance of reaction channels observed in a data sample that consisted of only about three hundred events. Forty five different reaction channels are listed. Clearly one cannot claim that all of these channels were definitely identified, but nonetheless the majority of them are surely present. It is worth reviewing that the analysis method which was employed did not put as many constraints on the data as it could have. For two-particle final states, coplanarity was not required. For $\pi^{\circ} \gamma$ and $\eta \gamma$ decays of omegas and phis, no attempt was made to require that the momenta of the odd photon and the massive particle matched the momentum of the decaying particle. The same statement also applies for $\eta^{\prime}$ decays to $\omega \gamma$. Nonetheless, one is struck by the cleanliness of the events in the Crystal Ball, and the fact that one can reconstruct events that had ten or more clusters. Unlike the kaon and pion data of the Crystal Ball, snakes are absent in the "good" antiproton events. Only one event in over 300 had a faint snake in it, and the clusters of that snake were removed by the analyzer's Crystal Ball TDC cut, leaving a perfectly-good, reconstructable antiproton-proton event. A sampling of SED pictures of several types of events is shown in Appendix B. 
Table 4: Table of reconstructed events found in the antiproton data analysis.

\begin{tabular}{|c|c|c|c|c|c|c|c|}
\hline Final & Run 927 & Run 928 & Run 929 & Run 930 & & Totals & \\
\hline State & Full Tgt & Full Tgt & Full Tgt & MT Tgt & "Crtn." & "Uncrtn." & Sum \\
\hline$\overline{\pi^{\circ} \pi^{\circ} \pi^{\circ}}$ & $666 \underline{6} 666$ & $(\overline{(1 \times \underline{6}, 9 \times 6)}$ & $(17 \times \underline{6}, 34 \times 6)$ & $\overline{6} 66$ & $\overline{48}$ & 21 & $\overline{69}$ \\
\hline$\eta^{\prime} \pi^{\circ} \pi^{\circ}$ & $\underline{10}$ & $\underline{8} \underline{8} \underline{8} \underline{8}$ & $\begin{array}{l}8 \underline{8} \underline{8} \underline{8} \frac{10}{8} \underline{10} \underline{6} \underline{6} \underline{8} 8 \\
\underline{8} 8 \underline{8} \underline{8}\end{array}$ & & 6 & 17 & 23 \\
\hline$\overline{K_{s} K_{s}}$ & & 8 & $(14 \times \underline{8}, 6 \times 8)$ & & 6 & 15 & 21 \\
\hline$\pi^{\circ} \pi^{\circ}$ & 4 & 4444 & $(1 \times \underline{4}, 12 \times 4)$ & & 16 & 2 & 18 \\
\hline$\omega \pi^{\circ} \pi^{\circ}$ & $\underline{7}$ & $7 \underline{7} \underline{7}$ & 77777777 & & 5 & 7 & 12 \\
\hline$\phi \pi^{\circ}$ & $\underline{5} 55$ & 5 & $55 \underline{5} 5 \underline{5} 5 \underline{5}$ & & 7 & 4 & 11 \\
\hline$\eta^{\prime} \omega$ & & $\underline{7}$ & $57 \underline{7} \underline{9} \underline{7} \underline{7} 59$ & & 4 & 6 & 10 \\
\hline$\eta \pi^{\circ}$ & & 4 & $\underline{44444448}$ & & 8 & 1 & 9 \\
\hline$\pi^{\circ} \pi^{\circ} \gamma$ & $\underline{5}$ & $\underline{5}$ & $5 \underline{5} \underline{5} 5555$ & & 5 & 4 & 9 \\
\hline$\omega \pi^{\circ}$ & 5 & $5 \underline{5}$ & $\underline{5} \underline{5} 55 \underline{5} \underline{5}$ & & 4 & 5 & 9 \\
\hline$\phi \eta^{\prime}$ & & & $\underline{7} \underline{7} \underline{7} 77 \underline{11} 7 \underline{7}$ & & 3 & 5 & 8 \\
\hline$\eta^{\prime} \eta$ & & $\underline{6} \underline{8}$ & $12 \underline{8} \underline{6} \underline{6} \underline{10} \underline{10}$ & & 1 & 7 & 8 \\
\hline$\omega \pi^{\circ} \pi^{\circ} \pi^{\circ}$ & & $\underline{9}$ & $\underline{9} \underline{9} \underline{9} \underline{9} \underline{9} \underline{9} 9$ & & 1 & 7 & 8 \\
\hline$\phi \pi^{\circ} \pi^{\circ}$ & 7 & 77 & $77 \underline{77}$ & & 6 & 1 & 7 \\
\hline$\eta^{\prime} \eta^{\prime}$ & & & $8108 \underline{8} 8 \underline{6}$ & & 4 & 2 & 6 \\
\hline$\eta^{\prime} \omega \pi^{\circ}$ & $\underline{9}$ & & $799 \underline{9} \underline{9}$ & & 3 & 3 & 6 \\
\hline$\overline{\pi^{\circ} \gamma \gamma}$ & 4 & 4 & $44 \underline{4}$ & & 4 & 1 & 5 \\
\hline$\eta \pi^{\circ} \pi^{\circ}$ & 6 & 6 & $6 \underline{6} \underline{6}$ & & 3 & 2 & 5 \\
\hline$\eta^{\prime} \pi^{\circ} \pi^{\circ} \pi^{\circ}$ & $\underline{10} \underline{8}$ & $\underline{10}$ & 1010 & & 0 & 5 & 5 \\
\hline$\phi \omega$ & & $\underline{6} 6$ & $\underline{6} 10$ & & 2 & 2 & 4 \\
\hline$\omega \omega$ & & & $6 \underline{6} 6$ & & 2 & 1 & 3 \\
\hline$\eta^{\prime} \gamma$ & & & $55 \underline{5}$ & & 2 & 1 & 3 \\
\hline$\omega \eta$ & 59 & $\underline{5}$ & & & 2 & 1 & 3 \\
\hline$\eta^{\prime} \pi^{\circ}$ & & & $\underline{6} \underline{6} 6$ & & 1 & 2 & 3 \\
\hline$\overline{\omega \gamma}$ & & & $\underline{4} \underline{4} 4$ & & 1 & 2 & 3 \\
\hline$\eta^{\prime} \eta \pi^{\circ}$ & & 8 & $\underline{8} \underline{10}$ & & 1 & 2 & 3 \\
\hline$\pi^{\circ} \pi^{\circ} \gamma \gamma \gamma$ & & & 77 & & 2 & 0 & 2 \\
\hline$\pi^{\circ} \pi^{\circ} \gamma \gamma$ & & & 66 & & 2 & 0 & 2 \\
\hline$\phi \pi^{\circ} \pi^{\circ} \pi^{\circ}$ & & & 99 & & 2 & 0 & 2 \\
\hline$\eta \eta$ & 4 & 4 & & & 2 & 0 & $\overline{2}$ \\
\hline$\phi \omega \pi^{\circ}$ & 8 & 8 & & & 2 & 0 & 2 \\
\hline$\eta \pi^{\circ} \pi^{\circ} \pi^{\circ}$ & & & $8 \underline{8}$ & & 1 & 1 & $\overline{2}$ \\
\hline$\pi^{\circ} \gamma \gamma \gamma$ & & & $5 \underline{5}$ & & 1 & 1 & 2 \\
\hline$\phi \eta \pi^{\circ}$ & 7 & 7 & & & 1 & 1 & 2 \\
\hline$\omega \eta \pi^{\circ}$ & & & $7 \underline{7}$ & & 1 & 1 & $\overline{2}$ \\
\hline$\phi \eta$ & & $\underline{9}$ & $\underline{5}$ & & 0 & 2 & 2 \\
\hline$\phi \gamma$ & & & 4 & & 1 & 0 & 1 \\
\hline$\gamma \gamma \gamma$ & & & 3 & & 1 & 0 & 1 \\
\hline$\omega \omega \pi^{\circ} \pi^{\circ}$ & & 10 & & & 1 & 0 & 1 \\
\hline $4 \pi^{\circ}$ & & 8 & & & 1 & 0 & 1 \\
\hline$\pi^{\circ} \gamma$ & & & $\underline{3}$ & & 0 & 1 & 1 \\
\hline$\eta \eta \pi^{\circ}$ & & & $\underline{10}$ & & 0 & 1 & 1 \\
\hline$\eta \pi^{\circ} \gamma$ & & $\underline{5}$ & & & 0 & 1 & 1 \\
\hline$\eta \eta \pi^{\circ} \pi^{\circ}$ & & & $\underline{8}$ & & 0 & 1 & 1 \\
\hline$\omega \omega \pi^{\circ}$ & & $\underline{8}$ & & & 0 & 1 & 1 \\
\hline Totals & 25 & 48 & 226 & 2 & 163 & 138 & 301 \\
\hline
\end{tabular}


Table 5: Breakdown of $4 \pi^{\circ}$ events in table 4 .

\begin{tabular}{|c||c|c|c|c|c||c|}
\hline Final State: & $K_{s} K_{s}$ & $\eta^{\prime} \pi^{\circ} \pi^{\circ}$ & $\eta^{\prime} \eta^{\prime}$ & $\eta \pi^{\circ}$ & $4 \pi^{\circ}$ (unique) & Total $4 \pi^{\circ}$ \\
\hline \hline Number of Events: & 23 & 11 & 1 & 1 & 1 & 37 \\
\hline
\end{tabular}

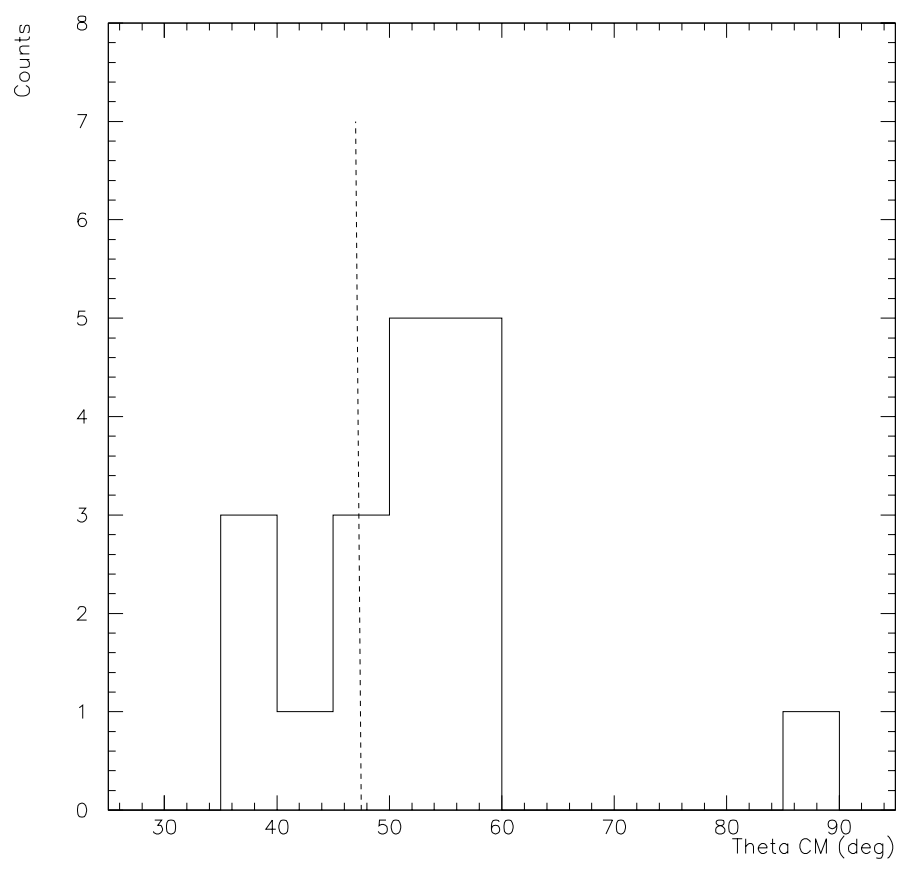

Figure 5: Distribution of $\theta_{C M}$ for observed $2 \pi^{\circ}$ events. The dashed line indicates the point at which the acceptance begins to be cut by the forward hole in the Crystal Ball.

It is clearly impossible to discuss each of the reactions listed in Table 4 in any detail in this document, however a few channels deserve to be discussed further. About $20 \%$ of the observed events were from the $3 \pi^{\circ}$ channel, making it by far the dominant reaction. Indeed, enough events were produced to attempt the crude Dalitz plot shown in Fig. 6 . One observes a clear signature of the $\mathrm{f}_{2}(1270)$ mesonic resonance, which was found to be prominant in this reaction channel by the Crystal Barrel experiment at LEAR.[1] There are a few channels that one would have to watch out for in terms of backgrounds to the $3 \pi^{\circ}$ channel. These include $2 \omega, \phi \omega, \eta^{\prime} \pi^{\circ}, \pi^{\circ} \pi^{\circ} \gamma \gamma$, and at slightly higher beam energies 


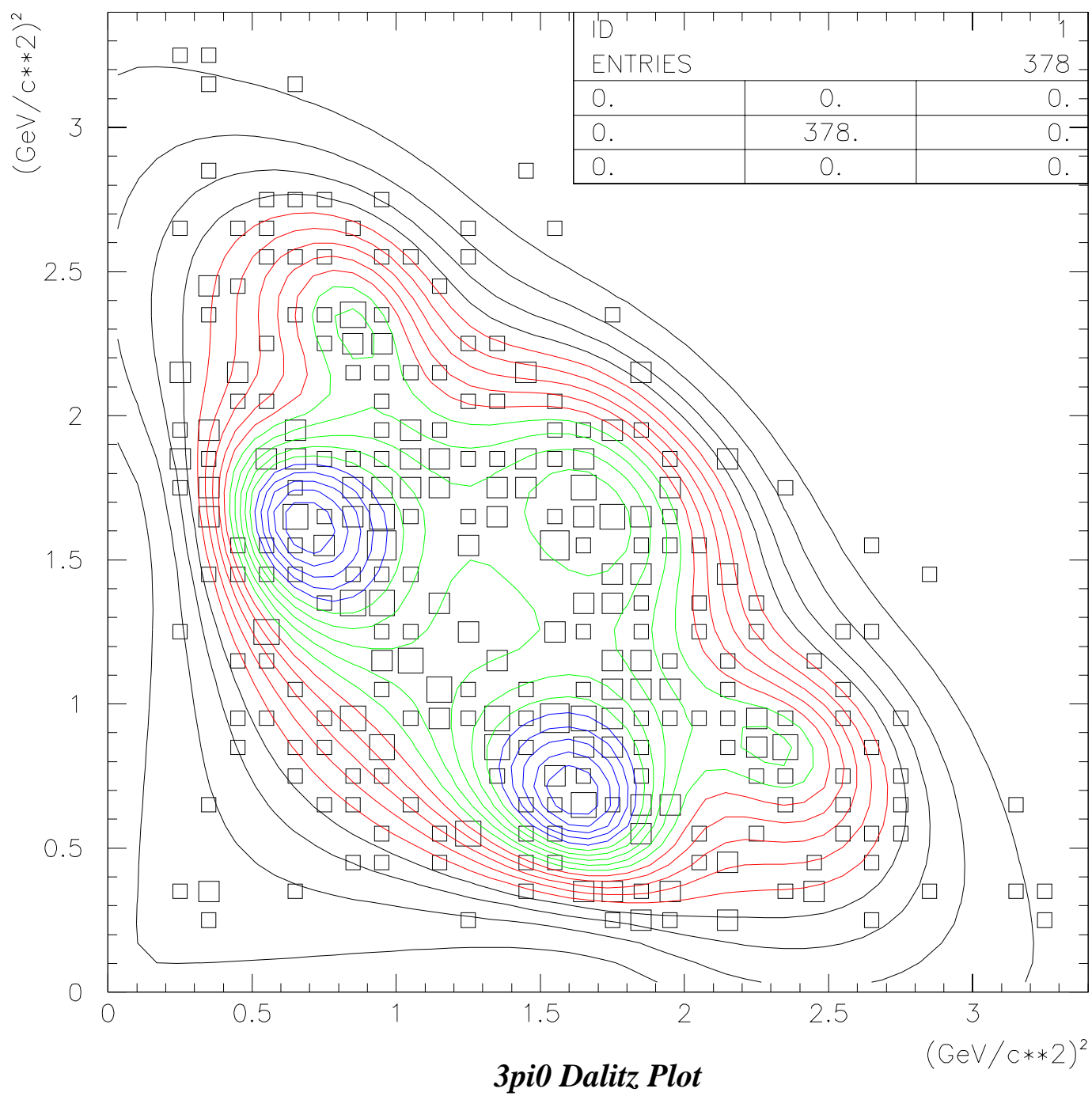

Figure 6: Dalitz Plot of the $3 \pi^{\circ}$ events. The two tall hills in the contours correspond to the $\mathrm{f}_{2}(1270)$ mesonic resonance, which dominates this channel. The reaction is $\bar{p}+p \rightarrow$ $\mathrm{f}_{2}(1270)+\pi^{\circ}$, where the $\mathrm{f}_{2}(1270)$ decays into two $\pi^{\circ}$ 's. There may also be hints of the $f_{0}(980)$ and the $f_{0}(1370)$, but the statistics are much too poor to tell with any certainty. (This plot was made from the 67 events that were observed in the three full-target runs 927-929.) 
the $2 \phi$ channel also will appear. The $2 \pi^{\circ}$ channel seemed to have a forward-peaked distribution, as Fig. 5 illustrates. Essentially all of the forward pions in the $2 \pi^{\circ}$ channel were near the downstream hole of the ball, suggesting that most of the forward pions from the $2 \pi^{\circ}$ reaction were missed entirely. Happily, at higher energies the cross section seems to broaden out more, so that one may perhaps do somewhat better at the energies available to the D6 line at the AGS. [2] A significant number of $\eta \pi^{\circ}$ events were observed, and they tend to be very clean and easy to identify. (This channel is of particular interest for charting $\mathrm{a}_{0}, \mathrm{a}_{2}$, and $\mathrm{a}_{4}$ mesonic resonances. In fact, the observed $\eta \pi^{\circ}$ events may have been produced largely through the $\mathrm{a}_{4}(2040)$.) The $\eta \pi^{\circ} \pi^{\circ}$ channel was seen and is another interesting one for finding lower-mass $\mathrm{a}_{0}$ resonances in Dalitz plots. The $4 \pi^{\circ} / 2 K_{s} / \eta^{\prime} \pi^{\circ} \pi^{\circ}$ group of channels represents a large chunk of the total neutral cross section. For reasons mentioned above, they are probably difficult to disentangle, however. The strong $\omega \pi^{\circ} \pi^{\circ}$ channel may be coming from the reaction $\bar{p}+p \rightarrow \mathrm{b}_{1}(1235)+\pi^{\circ}$, with the $\mathrm{b}_{1}(1235)$ decaying through its dominant mode of $\omega \pi^{\circ}$. Finally, the strong signal seen for $\phi \pi^{\circ}$ is a mystery. There are no resonances listed in the Particle Data Group tables that are known to decay to $\phi \pi^{\circ}$, and in general it was a surprise to see so many channels that involved $\phi$ production. (Interestingly, the LEAR Crystal Barrel Experiment also saw an unexpectedly large number of $\phi$ 's.[3])

Time did not permit such an analysis, but there is a class of neutral channels which should be obtainable in addition to the ones already listed. (See Appendix C). Instead of requiring the cut on the total energy in the ball, one could search for channels which have a missing mass of a $K_{L}$. Specifically, requiring four clusters with two $\pi^{\circ}$ 's that have a collective invariant mass of a $K_{S}$ would yield a $K_{L} K_{S}$ signal. This channel has long been known to have a structure in it at $600 \mathrm{MeV} / \mathrm{c}$ beam momentum indicating the existence of a resonance of mass $1970 \mathrm{MeV} / \mathrm{c}^{2}$ and a very narrow width of only $35 \mathrm{MeV}$.[4] Curiously, no such resonance is listed in the Particle Data Group Tables. Requiring an additional $\pi^{\circ}$ would give $K_{L} K_{S} \pi^{\circ}$, which must be fed strongly by the $\phi \pi^{\circ}$ channel. (Recall that this channel was already observed in Table 3 through what amount to rare decay modes of the $\phi$.) A Dalitz plot of this channel would also be interesting because there are a number of meson resonances that decay via $K \bar{K}$, and the channel 
$K_{S} K^{*}(892)^{\circ}$ should also be present.

In addition to analyzing the neutral triggers, a cursory attempt was made to see if one could find physics in the charged triggers. Specifically, three channels involving two-particle final states were looked for, namely $\bar{p} p$ elastic scattering, $\bar{p} p \rightarrow K^{+} K^{-}$, and $\bar{p} p \rightarrow \pi^{+} \pi^{-}$. Along with the same time of flight cut used for the neutral trigger analysis, two-cluster charged events were required. From these events one obtained the $\theta$ and $\phi$ angles of both clusters. From the azimuthal angles, a loose coplanarity cut was applied requiring that $\phi_{1}-\phi_{2}$ be within the range $140-220^{\circ}$. Using the values of $\theta_{1}$ and $\theta_{2}$ obtained from the two clusters, one could impose transverse and longitudinal momentum conservation to solve for the momenta $\left(p_{1}, p_{2}\right)$ of the two clusters. These were in turn used to calculate $\Delta E_{t o t}$, which was given by

$$
\Delta E_{t o t}=E_{i n c}-E_{1}-E_{2}
$$

where $E_{i n c}$ is the total energy of the initial system and $E_{i}=\sqrt{p_{i}^{2}+m^{2}}$. By inserting proton, kaon, or pion masses into the formulae for $E_{i}$ and looking for a peak near $\Delta E_{t o t}=0$, one could search for evidence of elastic scattering, $K^{+} K^{-}$production, or $\pi^{+} \pi^{-}$production. For run 929, the coplanarity histogram and the $\Delta E_{t o t}$ distributions for events which survived the coplanarity cut are shown in Fig. 7. Note that the energies of the two clusters are never used, only the angles.

In the data of Run 929, a clear elastic signal was observed, but nothing above background for the $K^{+} K^{-}$or the $\pi^{+} \pi^{-}$channels. The elastic peak from Run 929 contained about four hundred events. Scaling this by the counts in the beam scaler, one expected to see about seventy elastic events in Run 927 and ninety in Run 928. However, Runs 927 and 928 had only two and nine elastic events respectively. A glance at Table 1 provides the explanation - Run 929 had a CB total energy threshold setting of $1.0 \mathrm{~V}$ in the trigger electronics, and Runs 927 and 928 had an even higher threshold of $3.0 \mathrm{~V}$. Going from $1.0 \mathrm{~V}$ to $3.0 \mathrm{~V}$ in the threshold wiped out at least $90 \%$ of the elastic signal in the raw data of Runs 927 and 928. In the absence of data taken with lower CB thresholds, one cannot tell how much of the elastic signal was being lost even in Run 929. In addition, it is likely that the $1.0 \mathrm{~V}$ threshold of Run 929 might have killed the 

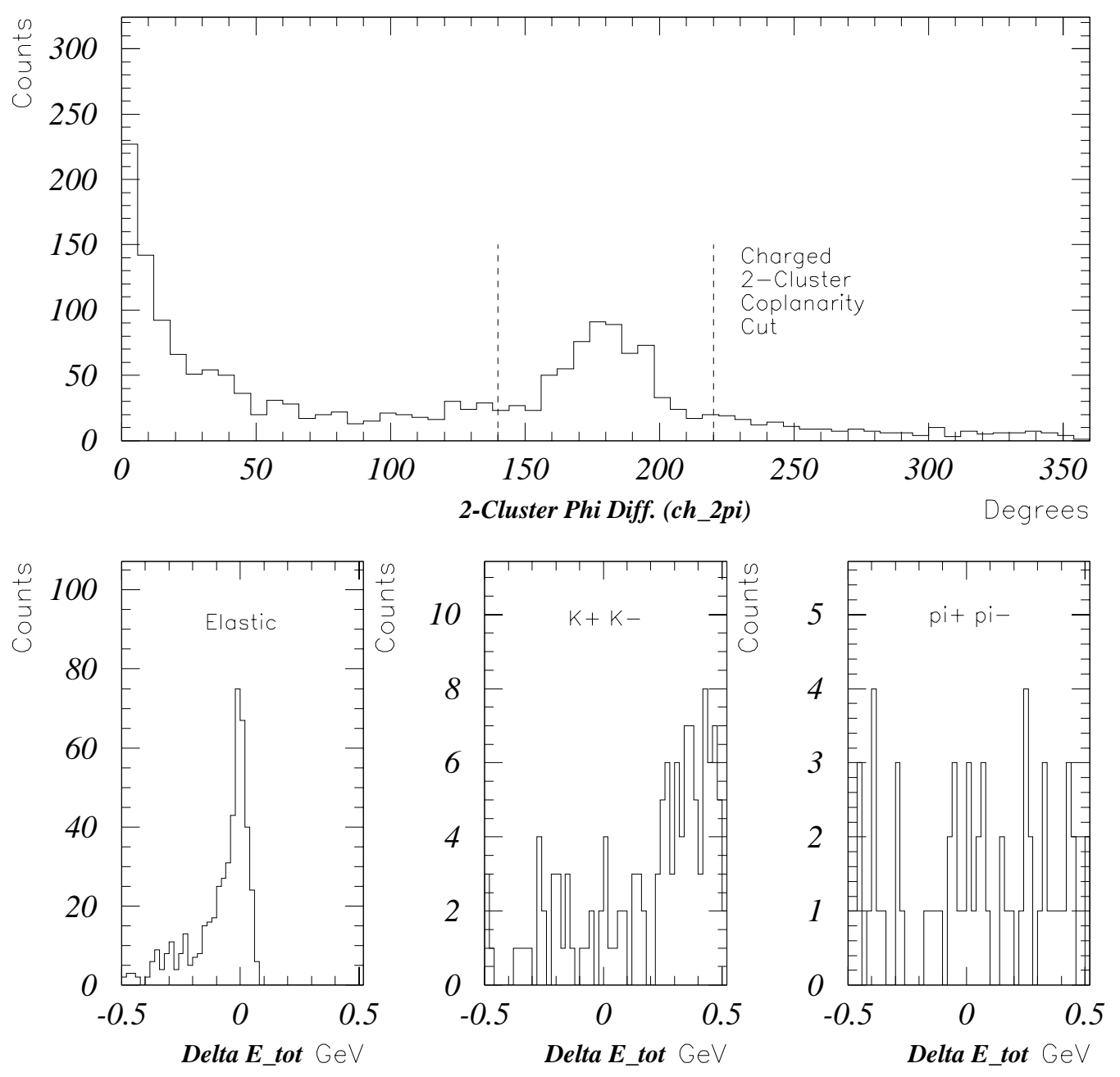

Figure 7: Histograms from analysis of charged trigger data from Run 929. Shown at top is the coplanarity histogram $\left(\phi_{1}-\phi_{2}\right)$. Below are the $\Delta E_{\text {tot }}$ histograms obtained by assuming elastic scattering, $K^{+} K^{-}$production, and $\pi^{+} \pi^{-}$production. A clear elastic signal was observed, but no evidence for the $K^{+} K^{-}$or $\pi^{+} \pi^{-}$channels above background. It is likely that all three channels were being cut out to varying degrees by the high $\mathrm{CB}$ total energy threshold setting (1.0V) that was being used in the trigger. Runs 927 and 928 had an even higher threshold setting of 3.0V, which resulted in a loss of at least $90 \%$ of the elastic signal that would have been expected based on the rate seen in Run 929 . 
signals for the $K^{+} K-$ and $\pi^{+} \pi^{-}$channels, since kaons and pions deposit less energy in the crystals via minimum ionization than protons and antiprotons do. In any case, one should remember to turn the CB energy threshold down in the trigger if one wishes to see physics in two-particle charged channels in future measurements! Obviously, having a charged particle tracker of the type envisioned for a Ke3 experiment would also be of great benefit for doing this kind of measurement. In particular, it would sharpen up the $\theta$ and $\phi$ determination (and hence the coplanarity) tremendously.

In theory at least, it should be possible to extract at least two other nuggets of physics from the charged data, namely $\pi^{\circ} \pi^{+} \pi^{-}$, and $\eta \pi^{+} \pi^{-}$. These are the charged versions of the $3 \pi^{\circ}$ and the $\eta \pi^{\circ} \pi^{\circ}$ channels seen in the neutral data, however they are augmented in the charged case by the addition of $\rho \pi^{\circ}$ and $\rho \eta$, neither of which appear in the neutral data because the rho always decays to $\pi^{+} \pi^{-}$and never to $2 \pi^{\circ}$. The $\rho \pi^{\circ}$ and $\rho \eta$ channels would be fed by the decays of $\pi^{*}, \omega^{*}$, and $\rho^{*}$ resonances.

Time did not permit a search for these channels, but the analysis would be similar to that for the "elastic" channels. One would first require 4-cluster events with one $\pi^{\circ}$ or one $\eta$, and perhaps supplement this with a check on whether the appropriate veto barrel elements fired based on the positons of the two odd clusters in the ball. At this point one would know the mass and momentum of the neutral particle $\overrightarrow{\mathbf{p}}_{\circ}$ and the angles of the other two clusters $\theta_{1}, \phi_{1}, \theta_{2}$, and $\phi_{2}$. Momentum conservation in $x, y$, and $z$ results in an overconstrained system of three equations in the two unknowns $p_{1}$ and $p_{2}$, which are the magnitudes of the momenta of the two charged particles. (One could solve for $p_{1}$ and $p_{2}$ in $x$ and $y$, and then cut on total $p_{z}$, perhaps.) From $p_{1}$ and $p_{2}$ and the assumption of pion masses for charged particles 1 and 2 , one can then calculate a $\Delta E_{t o t}$ as for the "elastic" case and apply a cut. Additional particle identification could be obtained from the energies of the charged clusters in the ball. Figure 8 shows the energy per cluster distributions of clusters from the charged events of run 929. One observes a clear minimum ionizing peak coming from charged pions which have enough energy to punch through the sodium iodide crystals. As one looks at events with more and more clusters, the average pion energy drops to the point where the minimum ionizing signal disappears, but it is clearly prominent in the four-cluster events. The Crystal Ball may 

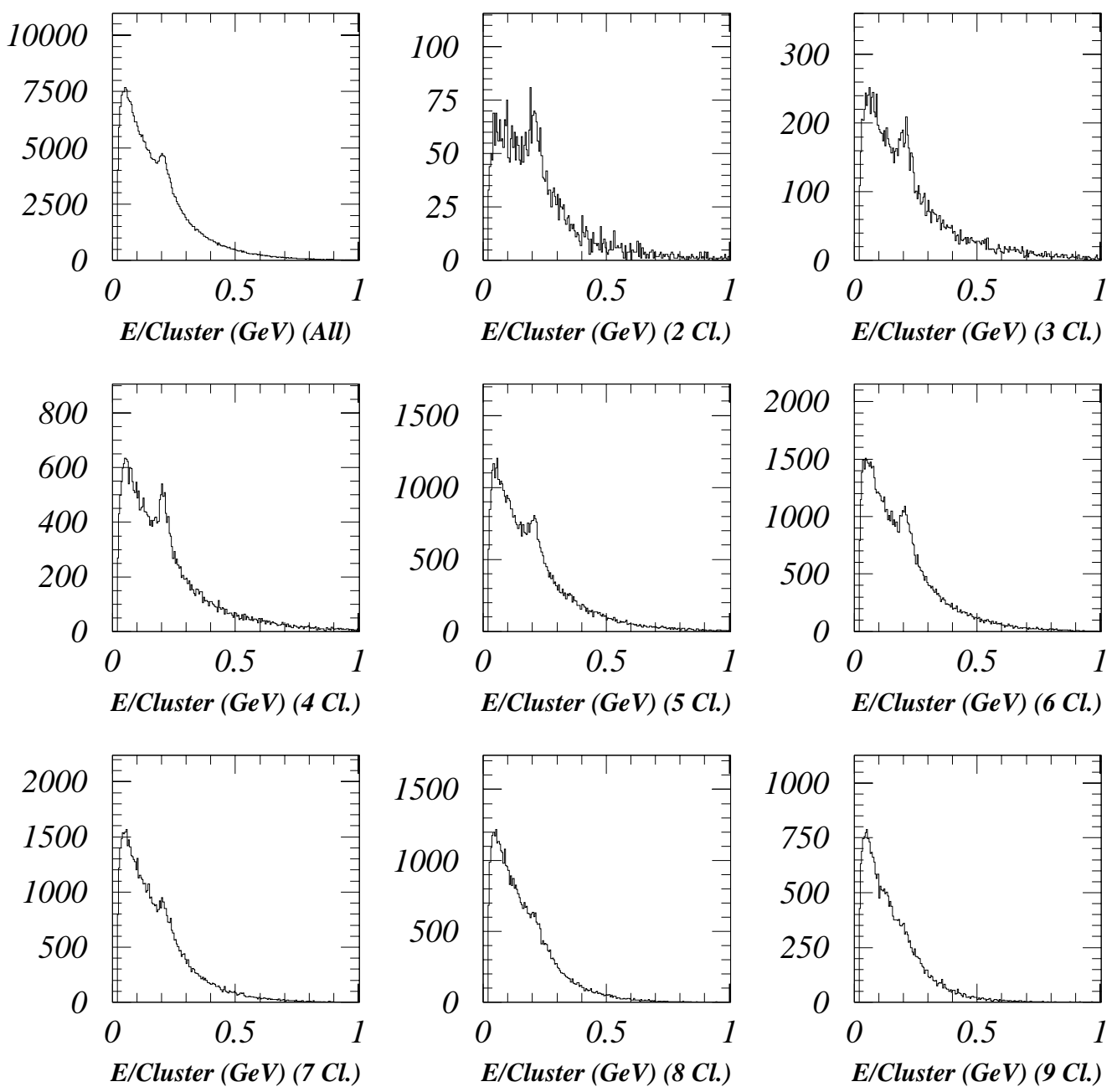

Figure 8: Energy per cluster histograms from charged events of various numbers of clusters in the data of Run 929. The upper left plot is for all events, and the other plots are for events with $2 \ldots 9$ clusters. The peak just above $200 \mathrm{MeV}$ comes from minimum ionizing particles, i.e. charged pions. As the number of clusters increases, the average energy of the charged pions drops until the pions no longer have enough energy to punch through the ball, at which point the minimum ionizing signal disappears. 
therefore be a rather useful device for charged pion identification. One could perhaps require that both of the charged clusters have energies consistent with being minimum ionizing particles. As long as one does not have to distinguish the $\pi^{+}$from the $\pi^{-}$among the two charged clusters, one should be able to obtain some nice physics from such an analysis. One would also expect a large cross section based on the rates observed for analogous three-particle neutral channels. Alternatively, one could assume Kaon masses for the two charged particles and look for the channel $K^{+} K^{-} \pi^{\circ}$. Again one would likely find the $\phi \pi^{\circ}$ channel, as $K^{+} K^{-}$is the favorite decay mode of the $\phi$. Also, one would expect to see extra $\pi^{\circ}$ 's coming from charged $K^{*}(892)$ decays. Comparing the $K^{+} K^{-} \pi^{\circ}$ and $K_{L} K_{S} \pi^{\circ}$ Dalitz plots might also be interesting, to see how different meson resonances contribute to each.

\section{Observed Rates and Cross Sections}

An attempt was made to determine the antiproton beam rate that was obtained during the running, as well as to calculate some rough total cross sections for the $3 \pi^{\circ}, 2 \pi^{\circ}$, and $\eta \pi^{\circ}$ reaction channels. The formula used for the cross section in units of millibarns was

$$
\sigma(\mathrm{mb})=\int \frac{d \sigma}{d \Omega} d \Omega=\frac{Y}{\left(N_{b}\right)\left(N_{t}\right)\left(10^{-27}\right)\left(\epsilon_{r e c}\right)(\Gamma)}
$$

where the individual terms are given by

$$
\begin{aligned}
Y & =\text { background-subtracted number of events observed, } \\
N_{b} & =\text { number of antiprotons that hit the target, } \\
N_{t} & =\text { number of protons in the target }\left(\text { in } \mathrm{cm}^{-2}\right) \\
10^{-27} & =\text { conversion factor from } \mathrm{cm}^{2} \text { to } \mathrm{mb} \\
\epsilon_{r e c} & =\text { reconstruction efficiency from Monte Carlo } \\
\Gamma & =\text { branching ratio for observed decay modes. }
\end{aligned}
$$


Aside from the conversion factor, each term requires further elaboration. Beginning with $N_{b}$, it was obtained from the formula

$$
N_{b}=(\text { counts in scaler } 106)\left(F_{[t o f]}\right)\left(F_{\left[x y_{-} c u t\right]}\right) .
$$

Scaler 106 was the beam normalization scaler. It counted the electronics coincidence $(S 1 \cdot S 2 \cdot S T) \cdot(\overline{H V}) \cdot(\overline{M B})$. Because this scaler did not discriminate between beam particle type (i.e. time of flight) or whether or not the particles actually hit the target, two correction factors appear in Eqn. 3. The factor $F_{[t o f]}$ is the fraction of antiprotons that came down the beam pipe as estimated from the S2 time of flight histogram. The factor $F_{\left[x y \_c u t\right]}$ is the fraction of events within the antiproton time of flight cut that are estimated to have hit the target based on tracking information from the upstream drift chambers. Note that absent the factor $F_{\left[x y_{-} c u t\right]}$ in Eqn. 3 one has the total number of antiprotons that came down the beamline irrespective of whether they hit the target. (Only events within the antiproton time of flight cut were used for the target vertex test in order to avoid possible problems with the pions having been less focused on the target than were the antiprotons.)

As shown in Fig. 1, the S1 to S2 time of flight spectrum had two peaks, one of which contained antiprotons and the other pions. (The number of kaons was negligible because the separators were set to accept antiprotons, thereby defocusing the kaons upstream of S2.) The time of flight window for antiprotons was between channels 50 and 200 in the spectrum, however the antiproton peak itself did not begin until well above channel 100. It was therefore decided to treat channels 50-100 as being representative of a constant background under the antiproton peak, and to estimate the number of antiprotons in the histogram by the expression

$$
\bar{p} \text { 's in S1-S2 tof histogram }=\sum_{50}^{200}(\mathrm{~S} 2 \mathrm{TDC})-3 \cdot \sum_{50}^{100}(\mathrm{~S} 2 \mathrm{TDC}) .
$$

The total number of events in the S2 histogram is given by the test chamber_event, so $F_{[t o f]}$ is given by 


$$
F_{[t o f]}=\frac{\sum_{50}^{200}(\mathrm{~S} 2 \mathrm{TDC})-3 \cdot \sum_{50}^{100}(\mathrm{~S} 2 \mathrm{TDC})}{\text { chamber_event }} .
$$

Another ratio of interest is the antiproton purity, defined as the fraction of antiprotons within the S2 time of flight cut:

$$
\bar{p} \text { purity }=\frac{\sum_{50}^{200}(\mathrm{~S} 2 \mathrm{TDC})-3 \cdot \sum_{50}^{100}(\mathrm{~S} 2 \mathrm{TDC})}{\sum_{50}^{200}(\mathrm{~S} 2 \mathrm{TDC})} .
$$

The target correction factor was calculated from the ratio

$$
F_{\left[x y \_c u t\right]}=\frac{x y c u t}{b m t r}
$$

where the tests bmtr and xycut were defined as shown below.

$$
\begin{aligned}
\text { bmtr }= & (\text { chamber_event }) \& \&(\text { unique_track_in_drift_chambers }) \& \& \\
& \left(\text { good_pbar_s } 2 \_t o f\right) \\
\text { sycut }= & (\text { bmtr }) \& \&(x y \text { vertex at tgt is } \mathrm{w} / \text { in the } 10 \mathrm{~cm} \text { diameter of tgt }) \\
\text { chamber_event }= & \text { Any trigger for which there was chamber information } \\
& \text { (i.e. neutral, beam, charged, elastic, or pip }) . \\
\text { good_pbar_s } 2 \_t o f= & \mathrm{S} 2 \text { TDC is in the range between channels } 50-200 .
\end{aligned}
$$

Table 6 shows the complete listing of ratios and the numbers of counts used to calculate them. The quantities are shown on a run-by-run basis and for the accumulated counts of runs 927-929. Also given are the numbers of antiprotons in the beam per spill and the numbers of antiprotons on the target per spill. Based on the statement written in the logbook during the running that there were about 5.2 teraprotons/spill of primary beam on the production target in the C6 line, the numbers of antiprotons in the beam and on target per $\mathrm{Tp}$ /spill are also given. (An error of $\pm 10 \%$ on the primary beam flux per spill was assumed.) Note that the values for the number of antiprotons in the beam are calculated for particles that made it through the collimators of the C6 beamline to the S2 and ST trigger counters, so the number of antiprotons produced at the C6 production target would be higher. However, during the antiproton running the collimators and slit 
Table 6: Table of numbers relating to antiproton beam flux.

\begin{tabular}{|c|c|c|c|c|c|c|}
\hline $\begin{array}{l}\text { Beam } \\
\text { Quantity }\end{array}$ & $\begin{array}{l}\text { Run } \\
927\end{array}$ & $\begin{array}{l}\text { Run } \\
928\end{array}$ & $\begin{array}{l}\text { Run } \\
929\end{array}$ & $\begin{array}{l}\sum \text { Runs } \\
927-929\end{array}$ & $\begin{array}{l}\text { Run } \\
930\end{array}$ & Comments \\
\hline \# of spills & 331 & 650 & 2869 & 3850 & 281 & (\# of scaler events) \\
\hline Scaler 106 & 273831 & 370576 & 1659779 & 2304186 & 431257 & Beam Norm. Scaler \\
\hline Sc.106/spill & $810 \pm 28$ & $770 \pm 28$ & $607 \pm 25$ & $649 \pm 25$ & $645 \pm 25$ & Tot. part.'s / spill \\
\hline$\sum_{50}^{100} \mathrm{~S} 2 \mathrm{TDC}$ & 20 & 33 & 817 & 870 & 168 & S2 tof background \\
\hline$\sum_{50}^{200} \mathrm{~S} 2 \mathrm{TDC}$ & 9039 & 14258 & 71928 & 95225 & 9005 & Sum in tof win. \\
\hline$\sum_{50}^{200}-3 \sum_{50}^{100}$ & 8979 & 14159 & 69477 & 92615 & 8501 & $\# \bar{p}$ 's in tof histo. \\
\hline cham_event & 15707 & 20488 & 86551 & 122746 & 12703 & \# chamber events \\
\hline$F_{[t o f]}$ & $\begin{array}{l}0.5716 \pm \\
0.0076\end{array}$ & $\begin{array}{l}0.6911 \pm \\
0.0076\end{array}$ & $\begin{array}{l}0.8027 \pm \\
0.0042\end{array}$ & $\begin{array}{l}0.7545 \pm \\
0.0034\end{array}$ & $\begin{array}{l}0.6693 \pm \\
0.0096\end{array}$ & (See Eqn. 5.) \\
\hline $\bar{p}$ purity & $\begin{array}{l}0.9934 \pm \\
0.0015\end{array}$ & $\begin{array}{l}0.9930 \pm \\
0.0012\end{array}$ & $\begin{array}{l}0.9659 \pm \\
0.0012\end{array}$ & $\begin{array}{c}0.97259 \pm \\
0.00093\end{array}$ & $\begin{array}{l}0.9440 \pm \\
0.0043\end{array}$ & (See Eqn. 6.) \\
\hline xycut & 331 & 650 & 2869 & 3850 & 281 & (See Eqn. 7.) \\
\hline$b m t r$ & 562 & 1026 & 4867 & 6455 & 582 & (See Eqn. 7.) \\
\hline$F_{\left[x y_{-} c u t\right]}$ & $\begin{array}{l}0.589 \pm \\
0.021\end{array}$ & $\begin{array}{l}0.633 \pm \\
0.015\end{array}$ & $\begin{array}{l}0.5895 \pm \\
0.0070\end{array}$ & $\begin{array}{l}0.5964 \pm \\
0.0061\end{array}$ & $\begin{array}{l}0.483 \pm \\
0.021\end{array}$ & (See Eqn. 7.) \\
\hline $\operatorname{Sc} 106 \cdot F_{[t o f]}$ & $\begin{array}{l}0.1565 \pm \\
0.0021\end{array}$ & $\begin{array}{l}0.2561 \pm \\
0.0028\end{array}$ & $\begin{array}{l}1.3323 \pm \\
0.0070\end{array}$ & $\begin{array}{l}1.7385 \pm \\
0.0079\end{array}$ & $\begin{array}{l}0.2886 \pm \\
0.0042\end{array}$ & $\begin{array}{l}\bar{p} \text { 's in beam } \\
\left(\times 10^{-6}\right)\end{array}$ \\
\hline$(\bar{p}$ 's bm. $) / \mathrm{sp}$ & $463 \pm 6$ & $532 \pm 6$ & $487 \pm 3$ & $489 \pm 2$ & $432 \pm 6$ & $\bar{p}$ 's in bm. / spill \\
\hline$(\bar{p} \mathrm{bm}) / \mathrm{Tp} / \mathrm{sp}$ & $89 \pm 9$ & $102 \pm 10$ & $94 \pm 9$ & $94 \pm 9$ & $83 \pm 8$ & $\bar{p}$ 's in bm./Tp/spill \\
\hline $\begin{array}{l}\mathrm{Sc} 106 \times F_{[t o f]} \\
\times F_{\left[x y_{-} c u t\right]} \\
\end{array}$ & $\begin{array}{l}0.0922 \pm \\
0.0017\end{array}$ & $\begin{array}{l}0.1621 \pm \\
0.0042\end{array}$ & $\begin{array}{l}0.7854 \pm \\
0.0102\end{array}$ & $\begin{array}{l}1.037 \pm \\
0.012\end{array}$ & $\begin{array}{l}0.1394 \pm \\
0.0064\end{array}$ & $\begin{array}{l}\bar{p} \text { 's in bm. on tgt. } \\
\left(\times 10^{-6}\right) \quad\left(\mathbf{N}_{\mathbf{b}}\right)\end{array}$ \\
\hline$(\bar{p}$ 's tg. $) / \mathrm{sp}$ & $273 \pm 5$ & $337 \pm 9$ & $287 \pm 4$ & $292 \pm 3$ & $209 \pm 9$ & $\bar{p}$ 's on tgt. / spill \\
\hline$(\bar{p} \operatorname{tg}) / \mathrm{Tp} / \mathrm{sp}$ & $52 \pm 5$ & $65 \pm 7$ & $55 \pm 5$ & $56 \pm 6$ & $40 \pm 4$ & $\bar{p}$ on tgt./Tp/spill \\
\hline
\end{tabular}


settings were left wide open to accept the maximum number of antiprotons, so the size of the beam that was counted (and triggered on) was defined by the (large) ST counter. The value of $N_{b}$ accumulated over runs $927-929$ was $1.037 \pm 0.012 \times 10^{6}$ antiprotons on target.

The quantity $N_{t}$ is given by

$$
N_{t}=(\rho)(t)\left(6.023 \times 10^{23}\right) /\left(M_{m o l}\right),
$$

where $\rho$ is the target density in $\mathrm{g} / \mathrm{cm}^{3}$, $\mathrm{t}$ is the effective target thickness, $6.023 \times 10^{23}$ is Avogadro's number, and $M_{m o l}$ is the mass per mole of the target material. The effective target thickness is given by the distance between the upstream and downstream limits of the bulges of the liquid hydrogen container multiplied by a geometric correction factor for the shape of the bulges. Table 7 shows the quantities used in calculating $N_{t}$. All quantities used in calculating $N_{t}$ were obtained from a kumac file which was used online for calculating $2 \pi^{\circ}$ cross sections during the kaon running in 1998. Uncertainties of $\pm 0.1 "$ and \pm 0.01 were assumed for the target thickness and the geometric correction, respectively.

Table 7: Table of quantities related to the calculation of $N_{t}$.

\begin{tabular}{|l||l|l|}
\hline Quantity & Value & Comment \\
\hline \hline$\rho$ & $0.0708 \mathrm{~g} / \mathrm{cm}^{3}$ & Density of $\mathrm{LH}_{2}$ \\
\hline$l$ & $4.16 \pm 0.10 \mathrm{in}$. & Target length \\
\hline$F_{\text {geom }}$ & $0.89 \pm 0.01$ & Geometric correction factor \\
\hline$t_{\text {eff }}$ & $9.40 \pm 0.25 \mathrm{~cm}$ & Effective target thickness \\
\hline$N_{a v}$ & $6.023 \pm 0.10 \times 10^{23} \mathrm{~mol}^{-1}$ & Avogadro's number \\
\hline$M_{m o l}$ & $1.00794 \mathrm{~g} / \mathrm{mol}$ & Mass $/$ mole \\
\hline \hline$N_{t}$ & $3.98 \pm 0.10 \times 10^{23} \mathrm{~cm}^{-2}$ & \\
\hline
\end{tabular}

The reconstruction efficiency $\epsilon_{\text {rec }}$ was calculated from Monte Carlo for the three reaction channels $3 \pi^{\circ}, 2 \pi^{\circ}$, and $\eta \pi^{\circ}$. Because these three reaction channels were relatively 
easy to identify in the analysis-by-hand event reconstruction method employed on the real data, it was assumed that any event with the right number of clusters in the ball and greater than $2.0 \mathrm{GeV}$ total energy deposited would have been correctly reconstructed as the appropriate type of event. (Time did not permit verification of this assumption, however.) Accordingly, events were thrown in Monte Carlo under the assumption of uniform phase space, and the reconstruction efficiency was simply given by

$$
\epsilon_{\text {rec }}=\frac{\left(\# \text { of events with } E_{t o t}>2.0 \mathrm{GeV} \text { in the Ball and right \# of clusters }\right)}{\text { (Total number of events thrown in Monte Carlo) }} .
$$

Aside from assuming perfect event identification efficiency, the assumption of uniform phase space is probably a bad one to make for attempting to calculate a total cross section, especially for the $2 \pi^{\circ}$ case, where the cross section is known to be forwardpeaked at this incident beam momentum.[2] It may well be that for the $2 \pi^{\circ}$ case, a large fraction of the total cross section is lost out the downstream hole of the Crystal Ball despite the fact that the ball is a detector with nearly-4 $\pi$ acceptance. This should be somewhat less of a problem for the $3 \pi^{\circ}$ and $\eta \pi^{\circ}$ channels, since there the clusters tend to be more widely distributed about the ball. Given the limited statistics for events observed in the real data, this rough method of calculating $\epsilon_{r e c}$ was believed to be adequate for the purpose.

The branching ratio factors in Eqn. 2 were calculated from the branching ratios for $\pi^{\circ} \rightarrow 2 \gamma$ and $\eta \rightarrow 2 \gamma$ given in the 1998 Particle Data Group Tables. The $\Gamma$ factors were given by $\left(\Gamma_{\pi^{\circ} \rightarrow 2 \gamma}\right)^{3},\left(\Gamma_{\pi^{\circ} \rightarrow 2 \gamma}\right)^{2}$, and $\left(\Gamma_{\pi^{\circ} \rightarrow 2 \gamma}\right) \cdot\left(\Gamma_{\eta \rightarrow 2 \gamma}\right)$, for the $3 \pi^{\circ}, 2 \pi^{\circ}$, and $\eta \pi^{\circ}$ channels, respectively.

Finally, the event yields from the real data were calculated for each category by adding the number of "certain" events in table 4 to half the number of "uncertain" events and subtracting the estimated number of background events from the walls of the hydrogen target container. This assumes that the uncertain events were correctly identified only half the time in the by-hand event reconstruction analysis. Only the $3 \pi^{\circ}$ channel had a significant number of uncertain events, and it is also likely to have had some background in it from other reaction channels (e.g. $2 \omega$ and $\eta 2 \pi^{\circ}$ ). The practice of including 
only half the number of uncertain events thus also constituted a background subtraction to a certain extent. Table 8 contains all the factors used in Eqn. 2 in attempting to calculate normalized cross sections for the three reaction channels mentioned above, as well as the total cross section results.

Table 8: Quantities in Eqn. 2 used for calculating total cross sections.

\begin{tabular}{|l||l||l||l|}
\hline Quantity & $\bar{p}+p \rightarrow 3 \pi^{\circ}$ & $\bar{p}+p \rightarrow 2 \pi^{\circ}$ & $\bar{p}+p \rightarrow \eta \pi^{\circ}$ \\
\hline \hline$Y_{\text {cert }}$ & 48 & 16 & 8 \\
\hline$\frac{1}{2} Y_{\text {uncert }}$ & 10.5 & 1 & 0.5 \\
\hline$Y_{\text {bkg }}$ & $2 \pm 1$ & $1 \pm 1$ & $1 \pm 1$ \\
\hline \hline$Y$ & $56.5 \pm 7.7$ & $16.0 \pm 4.2$ & $7.5 \pm 3.1$ \\
\hline \hline \multicolumn{5}{|||}{} & $(1.037 \pm 0.012) \times 10^{6}$ & $(1.037 \pm 0.012) \times 10^{6}$ & $(1.037 \pm 0.012) \times 10^{6}$ \\
\hline \hline$N_{b}$ & $(3.98 \pm 0.10) \times 10^{23}$ & $(3.98 \pm 0.10) \times 10^{23}$ & $(3.98 \pm 0.10) \times 10^{23}$ \\
\hline$N_{t}\left(\mathrm{~cm}{ }^{-2}\right)$ & $0.297 \pm 0.014$ & $0.449 \pm 0.016$ & $0.467 \pm 0.016$ \\
\hline$\epsilon_{\text {rec }}$ & $0.96437 \pm 0.00094$ & $0.97610 \pm 0.00064$ & $0.3874 \pm 0.0034$ \\
\hline$\Gamma$ & $0.465 \pm 0.069$ & $0.088 \pm 0.023$ & $0.100 \pm 0.042$ \\
\hline \hline$(\mathrm{mb})$ &
\end{tabular}

The value of $465 \pm 69 \mu \mathrm{b}$ for the $3 \pi^{\circ}$ cross section is high by about 1.4 standard deviations when compared with the results from the Crystal Barrel experiment. [1] (See Fig. 9.) Given the limited statistics and the crudeness of both the analysis and the methods used to estimate the cross section, one can be satisfied with the result. In any case one can see that all three channels have sizeable total cross sections in the vicinity of $100 \mu \mathrm{b}$ or more at $550 \mathrm{MeV} / \mathrm{c}$ incident beam momentum. Furthermore, one can conclude that essentially every reaction listed in Table 4 must have a cross section in excess of about $10 \mu \mathrm{b}$. 


\section{Previous Measurements}

There is only one antiproton experiment that has ever been done that was capable of doing the kind of multiphoton detection that $\bar{p} p$ annihilation into all-neutral final states requires, namely the Crystal Barrel experiment at the LEAR antiproton storage ring at CERN.[5] The CPLEAR experiment measured the channels $\pi^{+} K^{-} K^{\circ}$ and $\pi^{-} K^{+} \bar{K}^{\circ}$, and was therefore not designed to trigger on all-neutral multiphoton final states. [6, 7] A series of other LEAR experiments measured $\bar{p} p$ annihilation into a variety of twoparticle states. These include cross sections, differential cross sections, and even some spin observables for hyperon pair production, $\bar{n} n, \pi^{+} \pi^{-}, K^{+} K^{-}$, and elastic scattering (PS185,198,199,266,287 + others?). A representative selection of references for these results are Refs. [8, 9, 10, 11, 12, 13, 14]. Still other LEAR experiments focused on production of antihydrogen and antihelium and similar atomic physics with stopped antiprotons. LEAR was shut down in 1997 and the remaining functioning parts of it are now being used exclusively for atomic physics with antiprotons.

It is not an understatement to say that at least $90 \%$ of the Crystal Barrel measurements were done with stopped antiprotons. The prevailing mentality was to stop the antiprotons and measure every imaginable branching ratio with maximum statistics in order to study lower-mass meson resonances and to look for glueballs in 3-particle Dalitz plots, etc. Dozens of publications were produced and a comprehensive summary of the antiprotons-at-rest measurements is published as Ref. [3].

It was not until the last year of LEAR operation that serious measurements were done with antiprotons in flight. Consequently, data were only taken at fairly widelyspaced beam momenta between 600 and $1940 \mathrm{MeV} / \mathrm{c}$, as shown in Fig. 9. The statistics obtained were considerable. For example, the number of $3 \pi^{\circ}$ events obtained was in the range $1.2-6.0 \times 10^{5}$ at each beam momentum. The publications from the in-flight data have not yet all appeared in print, but several of them are already available.[1, 15, 16, 17, $18,19,20]$ The data for the $2 \pi^{\circ}$ channel have not yet been published, however preliminary data are available in Ref. [2]. The main point to be noted here is that the Crystal Barrel data are in the nature of a survey experiment because of the wide spacings between the 


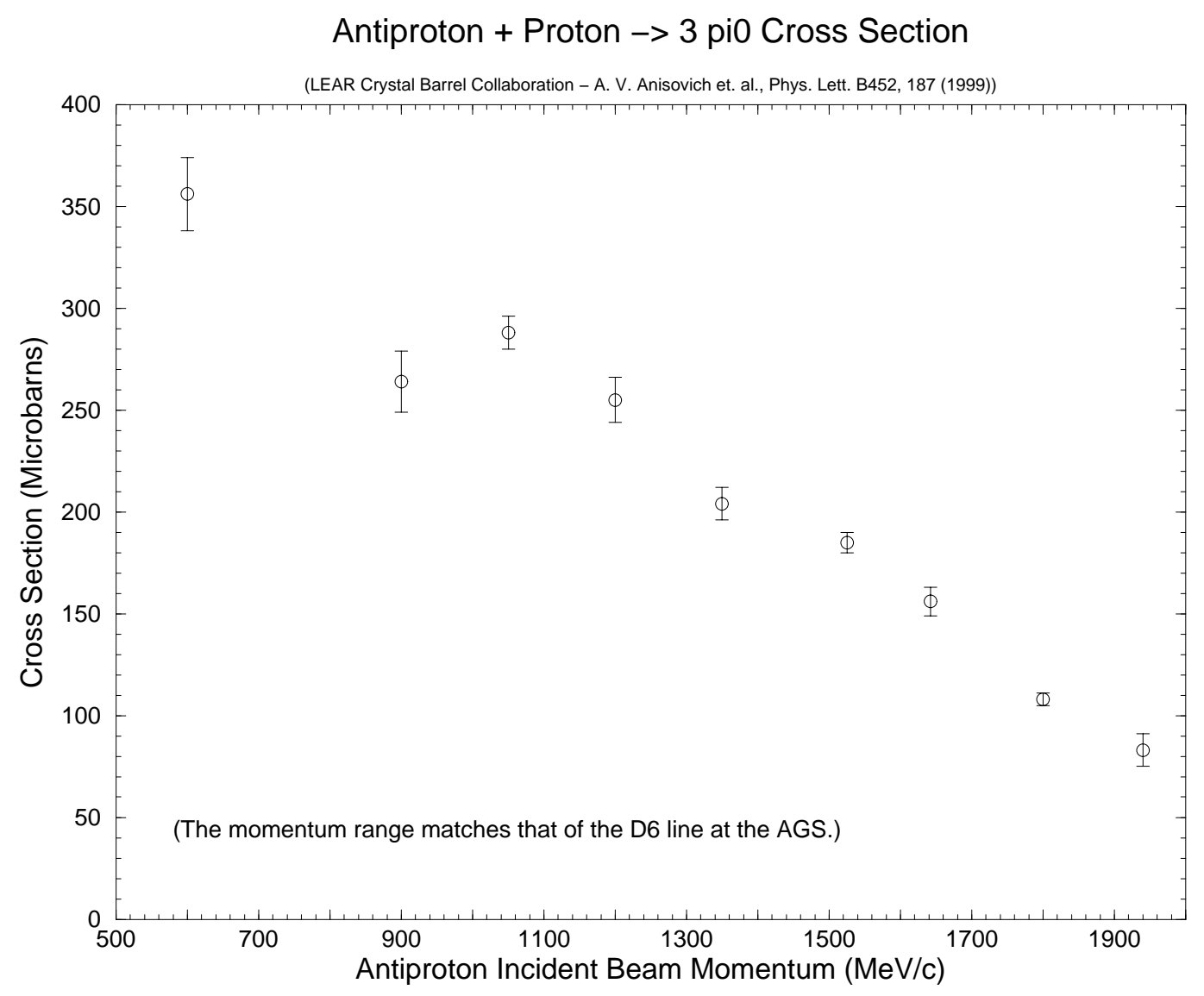

Figure 9: Crystal Barrel data for $\bar{p} p \rightarrow 3 \pi^{\circ}$ cross section as a function of beam momentum. [1] Points are fairly widely spaced in momentum. 
beam momenta that were chosen.

The only pre-LEAR references found for measurements of all-neutral final states from $\bar{p} p$ annihilation were two reports on $\bar{n} n$ total cross sections [21, 22] and one on $K_{L} K_{S}$ production,[4] although it may be that others exist. The literature also contains several older articles from bubble chamber experiments. References [23, 24, 25] are particularly interesting, as they claimed to have seen signals for some very narrow resonances in the $\pi^{+} \pi^{-} \pi^{+} \pi^{-} \pi^{\circ}$ channel and in elastic backscattering. There are of course numerous references for $\bar{p} p$ total elastic and total cross sections, for which current listings of world data can be obtained from the Particle Data Group's web page (http://www-pdg.lbl.gov).

\section{$5 \quad$ Physics Issues}

As the previous sections clearly show, there is enough physics to be gleaned from an antiproton experiment with the Crystal Ball to keep many people busy for years. However, because one of the aims of this document is to motivate and facilitate the writing of a proposal for doing an antiproton experiment with the Crystal Ball at the AGS, it is necessary to identify some overall physics goals that such an experiment would address. As such, one must find physics themes that will resonate with experimenters, review committees, and funding agencies while remaining within the limitations of what it will be possible to do at the AGS. This section is an attempt to suggest such themes. It is important to remember that the AGS is at this time probably the only place in the world where one can readily do medium-energy antiproton experiments.

Great strides in meson spectroscopy have been made in the past with $\bar{p} p$ annihilation experiments, but until recently relatively little has been done with the all-neutral final states because they require photon detection with nearly $4 \pi$ acceptance. At present, the non-strange meson resonances are poorly understood experimentally above masses of about $1300 \mathrm{MeV} / \mathrm{c}^{2}$. The evidence for the existence of many of the states in the Particle Data Group listings is not conclusive, and the listings themselves ignore many results that are in the literature. There is therefore a need for a more systematic program of measurements for the neutral mesons. The Crystal Ball is able to provide such 
measurements, and could do a lot to extend our knowledge.

Of particular interest in this respect is the emergence of enough experimental data to begin to see evidence for radial trajectories. The idea for radial trajectories was first put forward by Veneziano and Lovelace $[26,27]$ in 1968 , but it seems to have been long relegated to obscurity. The basic idea is that there are families of mesons that consist of successive radial excitations. The radial excitations within a family have the characteristic feature that the squares of the masses of the particles within a family are linear when plotted against the radial excitation quantum number $N=1,2,3, \ldots$. Furthermore, and most significantly, the theoretical argument predicts that the slopes of these "radial trajectories" are all the same regardless of which family of mesons one chooses. Figure 10 shows that it is now possible to identify what may be radial trajectories in several meson families. The trajectories are suprisingly linear, and the slopes are also in suprising agreement between families. This leads one to speculate whether, at least for mesons, all "true" $q \bar{q}$ resonances lie on radial trajectories, and those which do not have some origin outside the standard model such as dimeson bound states, glueballs, or other types of particles. If so, this would provide one with a radically new (old) idea for organizing the profusion of meson resonances that are to be found in nature. It would also challenge theorists as to why the highly nonlinear equations of QCD would give such a regular pattern of meson resonance masses. In fact, lattice QCD calculations are beginning to reach a level of sophistication that allows one to attempt to calculate meson resonance mass spectra. A better understanding of the experimental situation would help all concerned. It would be particularly important to do narrower momentum steps than were done by the Crystal Barrel so as to be sensitive to the possibility of narrow resonance states. While typical resonance widths are in the range 200-300 MeV, there are resonances that have widths narrower than $50 \mathrm{MeV}$ (e.g. $f_{J}(2220)$ ), and there may be others waiting to be found. 

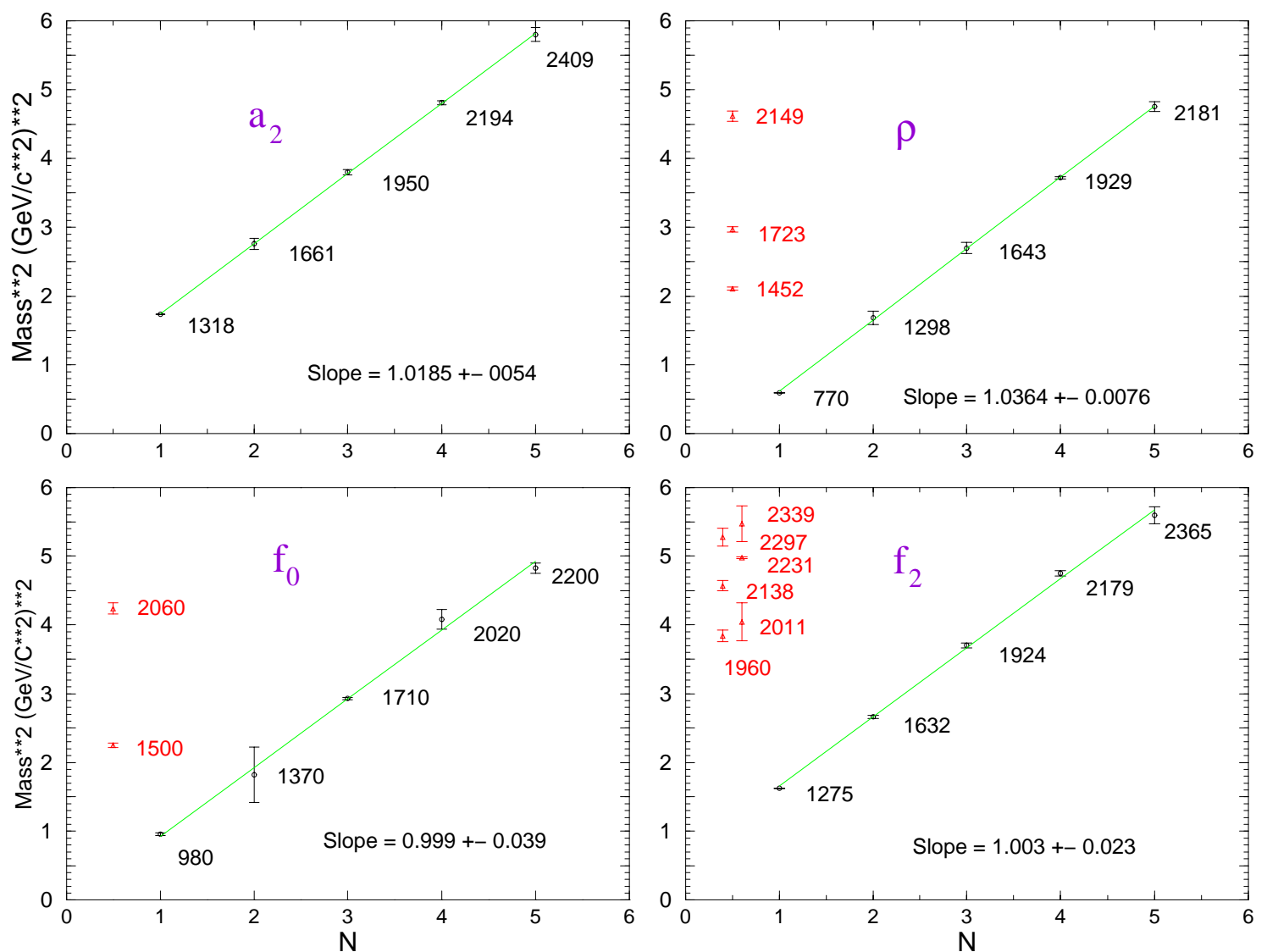

Figure 10: Some candidates for radial trajectories. The references for the individual data points come from [28] and from a recent Particle Data Group listing [29]. Many of the points that do not lie on trajectories are from the Particle Data Group tables. The evidence for many of these is not convincing. For others, the physics may simply be different, such as dimeson bound states or pure $s \bar{s}$ states, etc. In any case it would seem that they possess some fundamental difference(s) from the states on the trajories, despite the fact that all of the states have the same quantum numbers. This is interesting in itself, as the differences between the states on the trajectories and those not on the trajectories are really unknown. 
A well-designed antiproton experiment using the Crystal Ball at the AGS could provide a wealth of meson spectroscopy data, as each reaction channel can produce different types of resonances. Listed below are some of the resonances which contribute to various reaction channels.

$$
\begin{aligned}
& \bar{p} p \rightarrow 3 \pi^{\circ}: \quad \pi^{\circ}+f_{2}(1270), \quad \pi^{\circ}+f_{\circ}(980), \quad \pi^{\circ}+f_{\circ}(1370) \\
& \longleftrightarrow 2 \pi^{\circ} \quad \longleftrightarrow 2 \pi^{\circ} \quad \longleftrightarrow 2 \pi^{\circ} \\
& \bar{p} p \rightarrow 2 \pi^{\circ}: \quad f_{4}(2050), \quad f_{4}(2300), \quad f_{2}(2010) \\
& \longleftrightarrow 2 \pi^{\circ} \quad \longleftrightarrow 2 \pi^{\circ} \quad \longleftrightarrow 2 \pi^{\circ} \\
& \bar{p} p \rightarrow \eta \pi^{\circ}: \quad f_{4}(2050), \quad f_{2}(2010), \quad a_{4}(2040) \\
& \longleftrightarrow \eta \pi^{\circ} \quad \longleftrightarrow \eta \pi^{\circ} \quad \longleftrightarrow \eta \pi^{\circ} \\
& \begin{aligned}
& \bar{p} p \rightarrow 2 \pi^{\circ} \gamma: \quad \pi^{\circ} \omega, \pi^{\circ} \phi \\
& \longleftrightarrow \pi^{\circ} \gamma \quad \stackrel{\bigsqcup}{\longrightarrow} \pi^{\circ} \gamma
\end{aligned} \\
& \bar{p} p \rightarrow \eta \pi^{\circ} \pi^{\circ}: \quad \eta f_{2}(1270), \quad \pi^{\circ} a_{\circ}(980), \quad \pi^{\circ} a_{2}(1320) \\
& \longleftrightarrow 2 \pi^{\circ} \quad \longleftrightarrow \eta \pi^{\circ} \quad \longleftrightarrow \eta \pi^{\circ} \\
& \bar{p} p \rightarrow \eta \eta \pi^{\circ}: \quad \pi^{\circ} f_{2}^{\prime}(1525), \quad \pi^{\circ} f_{J}(1710) \\
& \longleftrightarrow 2 \eta \quad \longleftrightarrow 2 \eta \\
& \bar{p} p \rightarrow \omega \pi^{\circ} \pi^{\circ}: \quad \pi^{\circ} b_{1}(1235) \\
& \longleftrightarrow \pi^{\circ} \omega\left(\omega \rightarrow \pi^{\circ} \gamma\right) \\
& \bar{p} p \rightarrow 4 \pi^{\circ}: \quad \quad \pi^{\circ} \pi_{2}(1670) \\
& \longleftrightarrow 3 \pi^{\circ}
\end{aligned}
$$


In the likely event that one would be able to reconstruct final states with either two charged tracks or no charged tracks and a missing mass of a $K_{L}$, one would be in a position to identify kaon pairs. This would allow one to do extensive searches for strange decays of nonstrange mesons such as $X \rightarrow K \bar{K}, X \rightarrow \pi^{\circ} K \bar{K}, X \rightarrow K^{*} \bar{K}$, and others. Such information puts powerful constraints on theoretical models of meson structure. This information would also be complementary to similar measurements of strange decays of strange and nonstrange baryon resonances which the Crystal Ball could obtain from doing experiments with pion and kaon beams in the AGS D6 line.

There could also be opportunities to study rare and/or forbidden decays of various mesons in much the same way that has already been done for eta decays with the Crystal Ball. Decays of the $\rho, \omega, \eta^{\prime}$, and $\phi$ mesons would be available for study using the $\pi^{\circ} X$ reactions, provided one were to run with high beam intensity for high statistics at a single beam momentum for some time. Examples of interesting decays to look for might include forbidden $\rho$ decays into $2 \pi^{\circ}$ or $\pi^{\circ} \eta$, C-violating $\omega$ decays into $\eta \pi^{\circ}$ or $3 \pi^{\circ}$, C and $\mathrm{CP}$ violating $\eta^{\prime}$ decays into $\pi^{+} \pi^{-}, 2 \pi^{\circ}$, and $3 \gamma$, and a variety of $\phi$ decays, such as the P-violating mode $\omega \gamma$, the I and P-violating mode $\rho \gamma$, and the mode $\eta^{\prime} \gamma$, which must be nonzero because the $\eta \gamma$ mode is known to have a branching fraction in excess of $1 \%$. Many of these decay studies may require a tracker in the ball to identify pairs of charged pions.

\section{Experiment Plans for the AGS}

If one is going to propose an experiment with antiprotons at the AGS, one needs to set goals that one could reasonably achieve based on the available beam fluxes in AGS beamlines. Based on the single shift of antiproton data that was obtained in November 1998, one can conclude that in the C6 line low beam rates are a serious problem. As Table 6 indicates, the antiproton flux at $550 \mathrm{MeV} / \mathrm{c}$ was only about $100 / \mathrm{Tp} / \mathrm{spill}$, and it took about 300 minutes of beam time with $5.2 \mathrm{Tp} / \mathrm{spill}$ on the $\mathrm{C} 6$ production target to obtain an estimated $563 \pi^{\circ}$ events. Based on such running conditions, Table 9 shows the amounts of beam time that would be required to obtain $10003 \pi^{\circ}$ events at intervals of 
$50 \mathrm{MeV} / \mathrm{c}$ in incident momentum. The estimates assume that the antiproton flux scales as the fourth power of beam momentum[30], and the $3 \pi^{\circ}$ cross section data from the Crystal Barrel Experiment [1] (Fig. 9) were also used to adjust the estimates.

Table 9: Estimated running times for $10003 \pi^{\circ}$ events in the AGS C6 line.

\begin{tabular}{|c||c|c||c||c|}
\hline $\begin{array}{c}\mathrm{p} \\
(\mathrm{MeV} / \mathrm{c})\end{array}$ & $\begin{array}{c}\sigma_{3 \pi^{\circ}} \\
(\mu b)\end{array}$ & $\begin{array}{c}\text { Hrs. (const. } \sigma) \\
\left(p^{4} \text { scaling) }\right.\end{array}$ & $\begin{array}{c}\text { Hrs. }\left(10^{3} 3 \pi^{\circ} \text { 's) }\right. \\
(\text { with } \sigma \text { scaling })\end{array}$ & $\begin{array}{c}E_{C M} \\
(\mathrm{GeV})\end{array}$ \\
\hline \hline 450 & 402 & 199 & 184 & 1.950 \\
\hline 500 & 387 & 131 & 125 & 1.962 \\
\hline 550 & 371 & 89 & 89 & 1.975 \\
\hline 600 & 356 & 63 & 64 & 1.989 \\
\hline 650 & 341 & 46 & 50 & 2.004 \\
\hline 700 & 325 & 34 & 31 & 2.019 \\
\hline 750 & 310 & 26 & Total 582 Hrs. & \\
\hline \hline
\end{tabular}

One could imagine doing an experiment with 800-1000 hrs. of allocated beamtime to accomplish the program listed in Table 9. Such a data set would have barely adequate statistics to cover the $3 \pi^{\circ}$ Dalitz plots at each momentum, and adequate statistics for most other reaction channels would not have been obtained. Furthermore, the range of center of mass energies that the momentum range $450-750 \mathrm{MeV} / \mathrm{c}$ probes is very narrow and does not fully cover the full widths of typical resonances in this region (e.g. the $f_{4}(2050)$, which is the likely source of at least part of the $2 \pi^{\circ}$ signal). Although it might be interesting to attempt to reproduce the $K_{L} K_{S}$ results of Benvenuti et. al. [4], on the whole the C6 line is not the best location for doing antiproton physics at the AGS. It should be noted for comparison that the Crystal Barrel Experiment obtained about $200 \mathrm{k} 3 \pi^{\circ}$ events at each beam momentum during its antiprotons-in-flight measurements. Such statistics are clearly unobtainable in the C6 line, although one can debate about the statistics one would need to aim for depending on one's desired physics goals. 
Table 10: Estimated running times for $10003 \pi^{\circ}$ events in the AGS D6 line.

\begin{tabular}{|c|c|c|c|c|}
\hline $\begin{array}{c}\mathrm{p} \\
(\mathrm{MeV} / \mathrm{c})\end{array}$ & $\begin{array}{l}\sigma_{3 \pi^{\circ}} \\
(\mu b) \\
\end{array}$ & $\begin{array}{c}\text { Min. }(\text { const. } \sigma) \\
\left(p^{3.8} \text { scaling }\right)\end{array}$ & $\begin{array}{l}\text { Min. }\left(10^{3} 3 \pi^{\circ} \mathrm{s}\right) \\
\text { (with } \sigma \text { scaling) }\end{array}$ & $\begin{array}{c}E_{C M} \\
(\mathrm{GeV})\end{array}$ \\
\hline 550 & 371 & 430 & 430 & 1.950 \\
\hline 600 & 356 & 308 & 321 & 1.962 \\
\hline 650 & 335 & 227 & 251 & 1.975 \\
\hline 700 & 325 & 171 & 195 & 1.989 \\
\hline 750 & 300 & 131 & 162 & 2.004 \\
\hline 800 & 280 & 102 & 135 & 2.019 \\
\hline 850 & 270 & 81 & 111 & 2.034 \\
\hline 900 & 264 & 65 & 91 & 2.050 \\
\hline 950 & 275 & 53 & 71 & 2.065 \\
\hline 1000 & 285 & 43 & 56 & 2.083 \\
\hline 1050 & 288 & 36 & 46 & 2.098 \\
\hline 1100 & 285 & 30 & 39 & 2.115 \\
\hline 1150 & 270 & 25 & 34 & 2.132 \\
\hline 1200 & 255 & 22 & 32 & 2.149 \\
\hline 1250 & 240 & 18 & 28 & 2.166 \\
\hline 1300 & 225 & 16 & 26 & 2.184 \\
\hline 1350 & 204 & 14 & 25 & 2.201 \\
\hline 1400 & 200 & 12 & 22 & 2.219 \\
\hline 1450 & 190 & 10 & 19 & 2.236 \\
\hline 1500 & 187 & 9 & 18 & 2.254 \\
\hline 1550 & 180 & 8 & 16 & 2.272 \\
\hline 1600 & 160 & 7 & 16 & 2.289 \\
\hline 1650 & 156 & 6 & 14 & 2.307 \\
\hline 1700 & 140 & 6 & 16 & 2.325 \\
\hline 1750 & 125 & 5 & 15 & 2.342 \\
\hline 1800 & 108 & 4 & 14 & 2.360 \\
\hline 1850 & 100 & 4 & 15 & 2.378 \\
\hline 1900 & 90 & 4 & 16 & 2.395 \\
\hline 1950 & 83 & 3 & 13 & 2.413 \\
\hline
\end{tabular}


The prospects for antiprotons are likely to be much better in the D6 line. The D6 line's antiproton flux has been measured in the past at only 1.4 and $1.8 \mathrm{GeV} / \mathrm{c}$, so extrapolations of expected antiproton fluxes from those two mesurements are uncertain, but they indicate that the number of antiprotons/Tp/spill (at the end of the D6 line) is given by

$$
N(p)=(11.5 k)^{3.83},
$$

where $k$ is the momentum in $\mathrm{GeV} / \mathrm{c}$.[31] This indicates that the expected rate at 550 $\mathrm{MeV} / \mathrm{c}$ is $1169 \bar{p} / \mathrm{Tp} /$ spill in the D6 line, as compared to $94 \bar{p} / \mathrm{Tp} /$ spill as seen in Table 6 . Based on this estimate, one would expect to obtain $10003 \pi^{\circ}$ events in only 430 minutes, rather than the 5340 minutes as indicated in Table 9. Table 10 contains estimates for running times in the D6 line to obtain $10003 \pi^{\circ}$ events, assuming that $100 \%$ of the beam hits the target. The times are typically only a few minutes rather than the few hours typical of the C6 line. Furthermore, the momentum range stretches from at least 550 $\mathrm{MeV} / \mathrm{c}$ at the low end to about $1900 \mathrm{MeV} / \mathrm{c}$ at the high end, thus providing about the same momentum range as was avaliable to LEAR experiments. The entire program outlined in Table 10 would take less than 40 hours of running time, assuming again 5.2 $\mathrm{Tp} /$ spill of primary beam incident on the D6 production target. It is highly likely that one could expect to achieve at least twice that rate on the D6 target, since the C6 line only receives primary beam left over from other experiments upstream of it. Certainly one would wish to run the beamline at maximum intensity for antiproton running at all times to get sufficient statistics. The estimates in Table 10 may well be on the optimistic side, and it would be wise to perhaps ask for a short test run to better determine the antiproton fluxes that the D6 line is capable of, but even with pessimistic assumptions one expects much higher rates and in any case much greater momentum range in the D6 line. Unlike the C6 line, the momentum range in the D6 line is quite adequate to cover the full widths of many known meson resonances, and the statistics one could obtain would also be much better. It is difficult to imagine that one could ultimately compete with the massive statistics obtained by the Crystal Ball Experiment, but one should be able to do a lot of good physics with smaller momentum steps than were done in the 
Crystal Barrel in-flight measurement program. If the estimates in Table 10 hold true and one can obtain higher than 5.2 Tp of primary beam on the D6 target one can imagine obtaining several times $10^{4} 3 \pi^{\circ}$ events at each beam momentum in 2000-3000 hours of running time. Most likely $10^{5} 3 \pi^{\circ}$ events is a rough upper limit to the kind of statistics one could obtain, but that is quite sufficient for most physics purposes. It is not in general necessary to match the statistics that the Crystal Ball obtained.

Table 10 also indicates that the rates could be quite high at the upper end of the momentum range. In the $\mathrm{C} 6$ line, about half the incident beam consisted of pions despite the fact that the beam was tuned for antiprotons, and the situation would probably be worse at higher momenta. A chance conversation at BNL with Thomas Roser (AGS deputy director) revealed that it may be possible to clean up the antiproton beam in the D6 line by trying a new extraction technique, and that furthermore trying out this technique would be of great interest to the AGS machine operators as well. The method might also work for kaons, at least at lower momenta. The idea is as follows: First one extracts protons from the AGS with a microstructure of small bunches having durations of a few nanoseconds and spaced apart by a millisecond or more. These bunches are brought to the D6 production target where they produce pions, kaons, and antiprotons. At some (far) distance down the D6 beamline one installs a fast kicker magnet that is timed to deflect the pions (which arrive some nanoseconds ahead of the antiprotons) while leaving the antiprotons undisturbed. The result is a nearly perfectly clean antiproton beam at the D6 experimental area. Indeed, one can see how clean from the values in Table 6 of the " $\bar{p}$ purity" obtained from the data in the C6 line. (See also Eqn. 6.) This technique may allow one to obtain very clean high-intensity antiproton (or kaon) beams in the D6 line, which one would especially want if one wished to do rare decay physics with $\rho, \omega, \eta^{\prime}$, or $\phi$ mesons.

\section{Conclusions}

The test data taken with an antiproton beam tune during the 1998 Crystal Ball running period show that it is feasible to do antiproton physics with the Crystal Ball at the AGS. 
The data obtained, while not of sufficient statistics to warrant publication, indicate that there is tremendous physics potential to be tapped by doing such measurements and that the Crystal Ball is a great instrument for the purpose. There are probably more than 50 different charged and neutral proton-antiproton annihilation reactions for which data can be taken simultaneously, most of which do not require any modifications to the existing Crystal Ball hardware at all. The addition of a tracker for the Ke3 experiment will, however, enhance the antiproton capabilities of the Ball as well. Many of the reaction channels have cross sections in excess of $100 \mu \mathrm{b}$, and the rates obtainable for antiprotons are sufficient for doing good physics at the AGS. There is only one experiment that has ever attempted to do the same physics that the Crystal Ball could do, namely the Crystal Barrel experiment at LEAR. However, the Crystal Barrel experiment neglected to do very much with antiprotons in flight, thus leaving considerable room for the Crystal Ball to improve and extend upon their work. While antiproton physics is possible in the C6 line, the D6 line would be strongly preferred both for its better beam intensities and its greater momentum range.

The physics which a medium-energy antiproton-proton experiment with the Crystal Ball at the AGS could address would fall under at least three categories, namely 1) meson spectroscopy with an aim to perhaps helping to revive the idea of radial trajectories in meson resonances, 2) observation of strange decays of nonstrange mesons (complementary to measurements of strange decays of strange and nonstrange baryons which could be done with the same experimental setup but with the beamline tuned for pions or kaons instead of antiprotons), and 3) rare decays of the $\omega, \rho, \eta^{\prime}$, and $\phi$ mesons with a view toward testing Charge, Parity, Isospin, and other symmetries.

In short, the Crystal Ball Collaboration should entertain the idea of writing a proposal for doing antiproton physics with the D6 line in mind. Such a proposal could be logically coupled to an overall program that would also do measurements with pion and kaon beams. A program of this type would use the meson beams to study baryon resonances and antiprotons to study meson resonances, thereby covering both types of hadron physics at medium energies. 


\section{References}

[1] A. V. Anisovich et. al., Phys. Lett. B 452, 187 (1999).

[2] D. V. Bugg and A. Sarantsev, Crystal Barrel Note 337 (1998). (Obtainable from the Crystal Barrel web site http://www.phys.cmu.edu/cb_cern.html. This is a draft of an article that will most likely be published soon.)

[3] C. Amsler, Rev. Mod. Phys. 70, 1293 (1998).

[4] A. Benvenuti et. al., Phys. Rev. Lett. 27, 283 (1971).

[5] E. Aker et. al., Nucl. Instr. Meth. A321, 69 (1992).

[6] R. Adler et. al., Nucl. Instr. Meth. A379, 76 (1996).

[7] R. Adler et. al., Nucl. Instr. Meth. A390, 293 (1997).

[8] R. Birsa et. al., Phys. Lett. B 246, 267 (1990).

[9] R. Birsa et. al., Phys. Lett. B 273, 533 (1991).

[10] R. Birsa et. al., Nucl. Phys. B403, 25 (1993).

[11] A. Hasan et. al., Nucl. Phys. B378, 3 (1992).

[12] R. A. Kunne et. al., Nucl. Phys. B323, 1 (1989).

[13] F. Perrot-Kunne et. al., Phys. Lett. B 261, 188 (1991).

[14] R. A. Kunne et. al., Phys. Lett. B 261, 191 (1991).

[15] A. Abele et. al., Eur. Phys. J. C 8, 67 (1999).

[16] A. V. Anisovich et. al., Phys. Lett. B 452, 173 (1999).

[17] A. V. Anisovich et. al., Phys. Lett. B 449, 145 (1999).

[18] C. A. Baker et. al., Phys. Lett. B 449, 114 (1999). 
[19] A. V. Anisovich et. al., Phys. Lett. B 452, 180 (1999).

[20] A. V. Anisovich et. al., Phys. Lett. B 449, 154 (1999).

[21] M. Alston-Garnjost et. al., Phys. Rev. Lett. 35, 1685 (1975).

[22] R. P. Hamilton et. al., Phys. Rev. Lett. 44, 1179 (1980).

[23] C. DeFoix et. al., Nucl. Phys. 162, 12 (1980).

[24] D. Cline et. al., Phys. Rev. Lett. 21, 1268 (1968).

[25] Ch. D’Andlau et. al., Phys. Lett. 58B, 223 (1975).

[26] G. Veneziano, Nouv. Cim. 57, 190 (1968).

[27] C. Lovelace, Phys. Lett. 28B, 264 (1968).

[28] D. C. Peaslee, Presentation to Workshop on Hadron Spectroscopy 99, Frascati, Italy, Mar. 8-12,1999.

[29] Particle Data Group, Eur. Phys. J. C 3, 1 (1998).

[30] P. Pile, AGS Beamline expert, numerous private communications.

[31] D. Peaslee, report entitled "Antiprotons in the D6 Line at BNL," based on extensive communication with Phil Pile and other sources. 


\section{Appendix A Source Codes of Analysis Routines}

In the hopes of sparing people some amount of work in the future (in case we ever do some real antiproton running), the source codes of the routines used in the antiproton analysis are given here. The routines are not suitable for analyzing large numbers of events, nor were they written with efficiency in mind, but they may prove useful as starting points for future efforts. The source codes below can be lifted directly out of the TeX file of this CB note.

The implementation within the analyzer of subroutine inv_miss_all used the following include file:

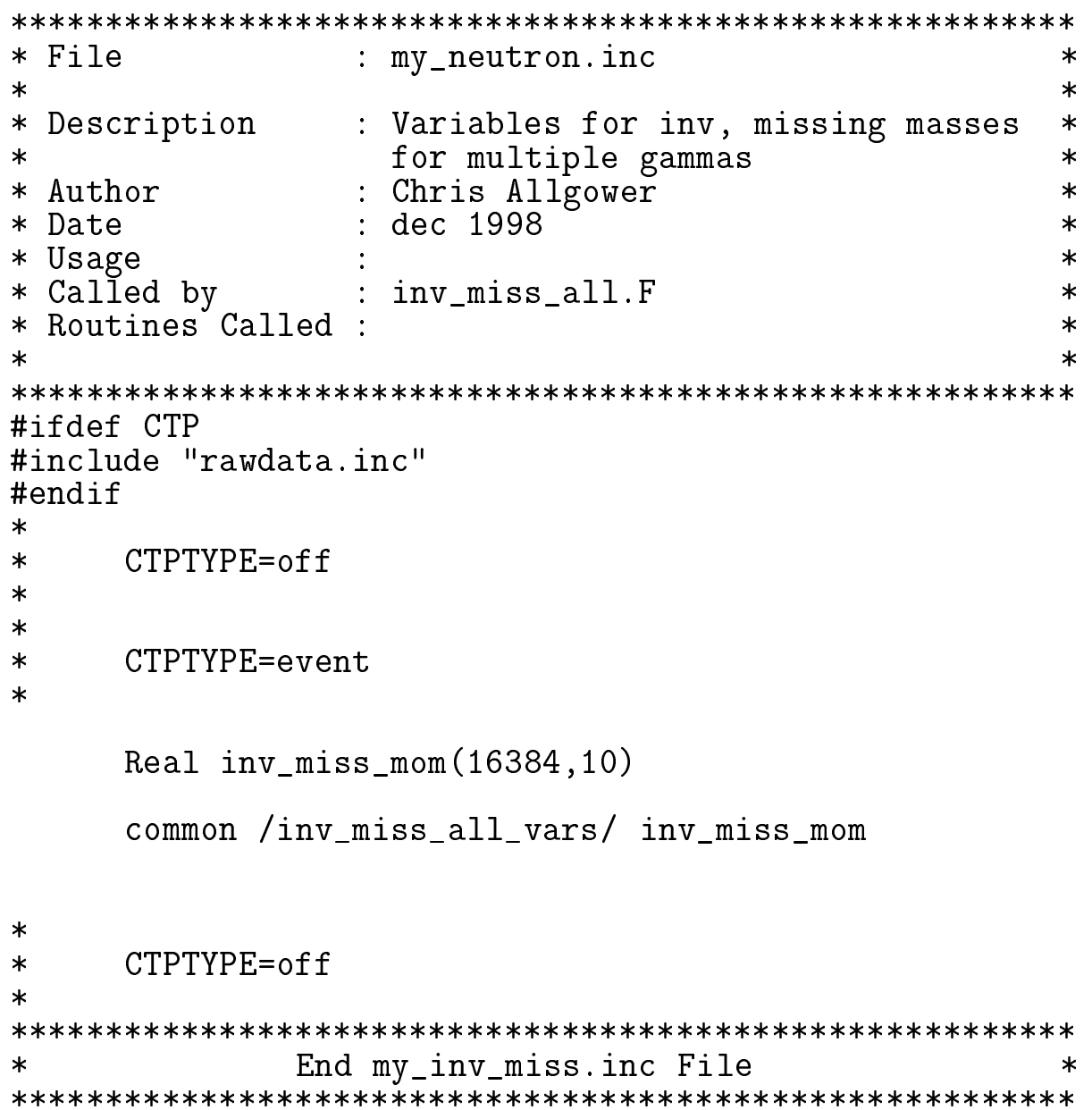


The source code for subroutine inv_miss_all is given below. It was called from subroutine usr_event. The routine writes all its information to IO unit 37, which is an ascii data file called inv_miss.dat. (The data file was opened in routine usr_ini and closed in usr_end.) In practice, inv_miss.dat could rapidly get to be a very large file, since for each event $2^{\text {(number of clusters) }}$ lines of text are written, each of which contains eleven numbers. For example, for the 250 "good" events of Run 929, the inv_miss.dat file exceeded 5 Mbytes in size. For the few events that were being analyzed, this method did the job. However, future implementations for doing serious analysis of antiproton data will need to find a better way to handle this aspect of the problem.

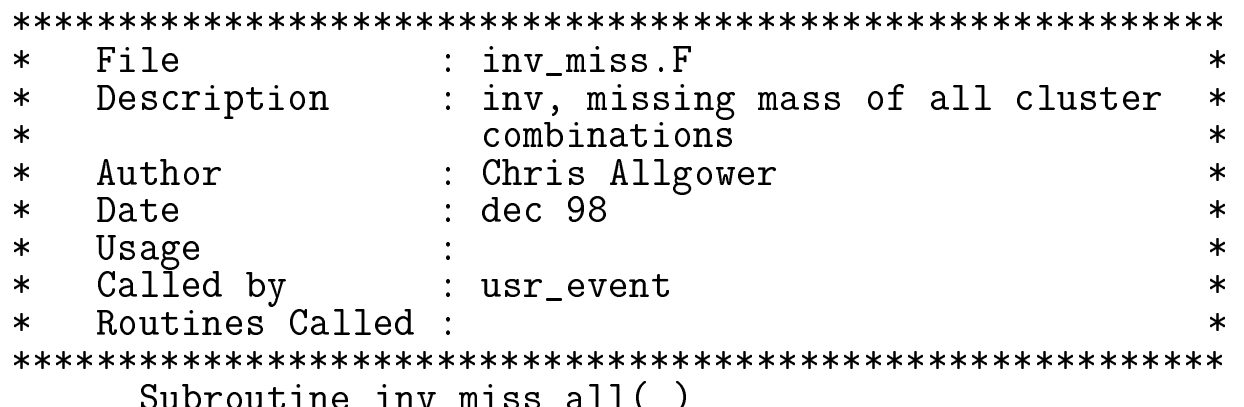

Implicit None

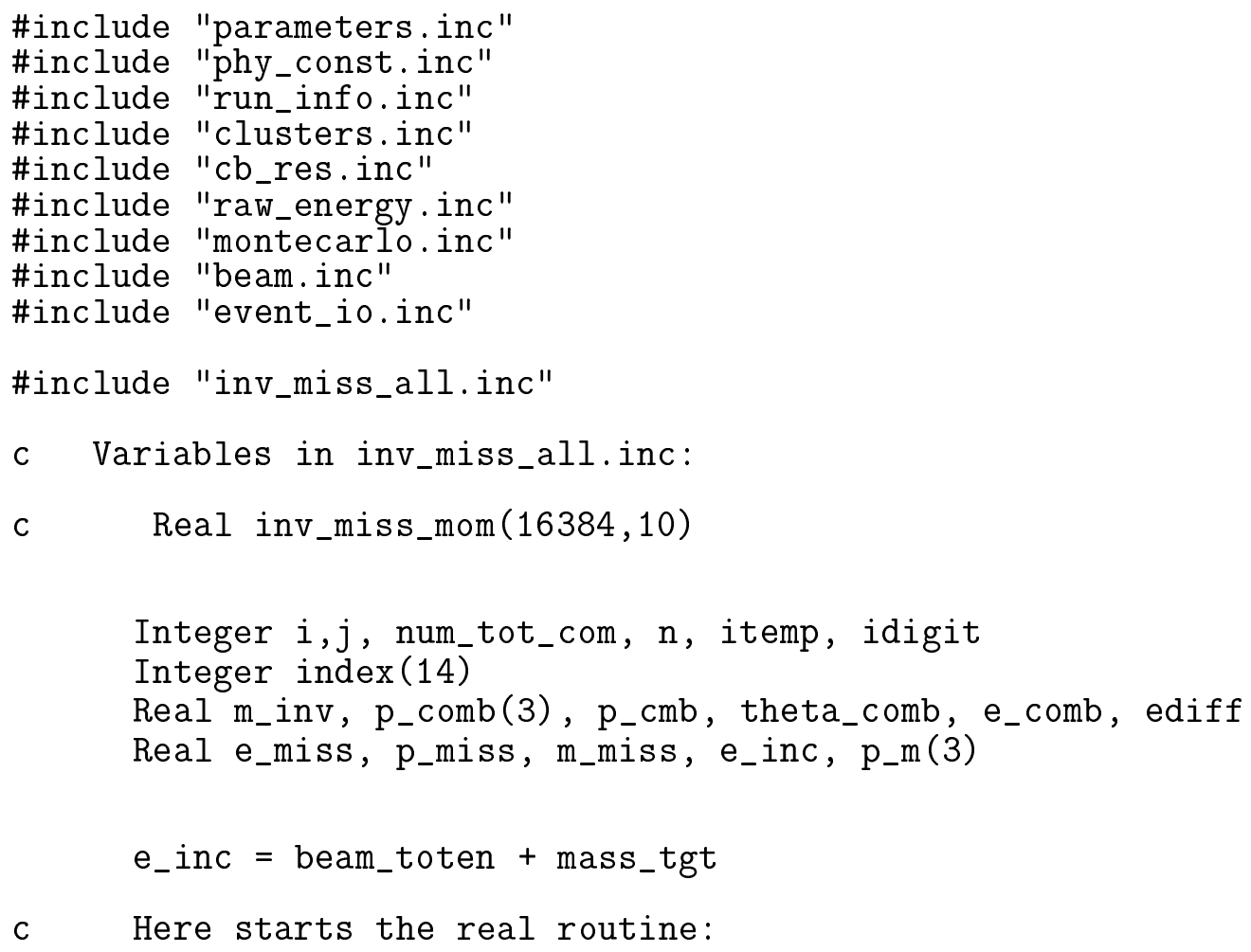




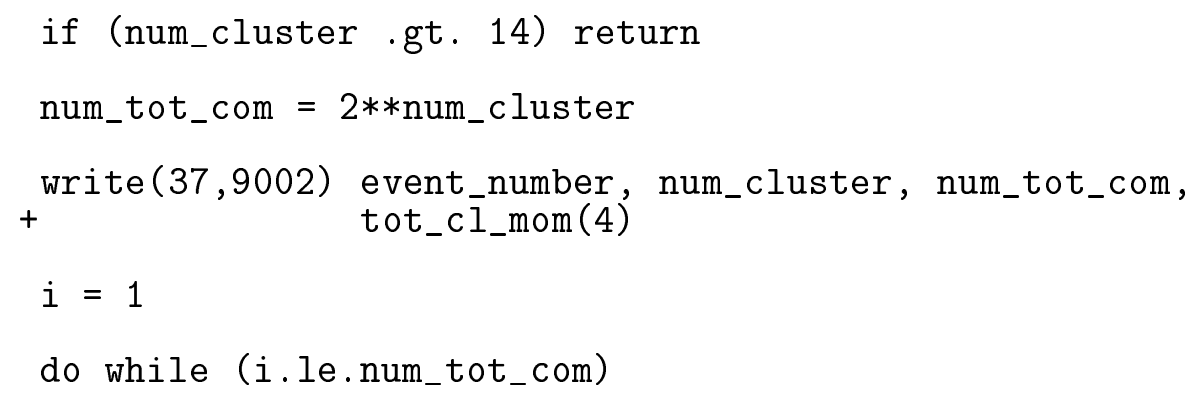


$p_{\text {_comb }}(2)=0.0$
$p_{-} \operatorname{comb}(3)=0.0$
$e_{-} \operatorname{comb}=0.0$

if (n.eq.1) then

C

Case for single cluster:

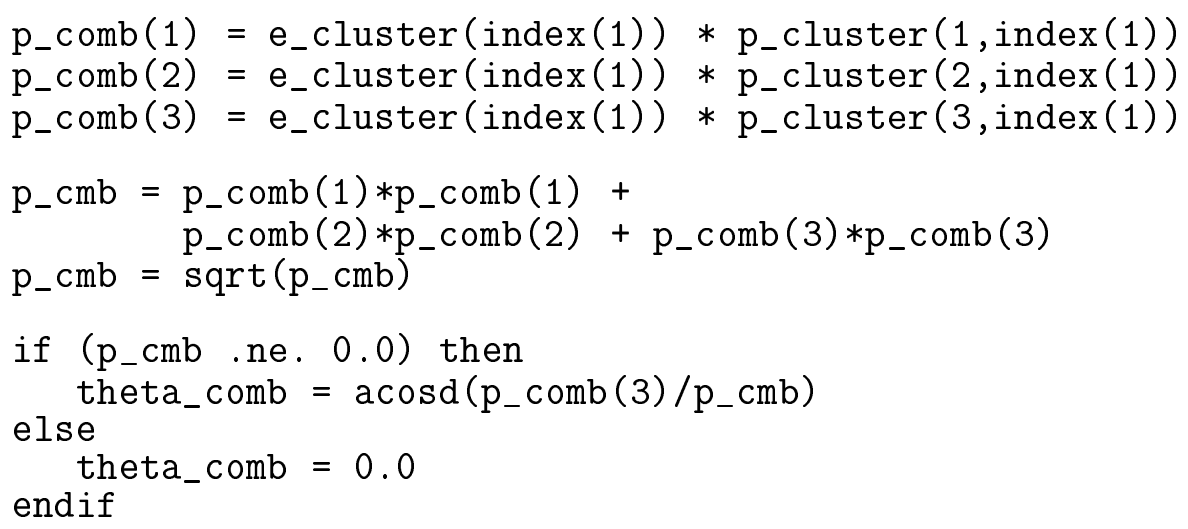




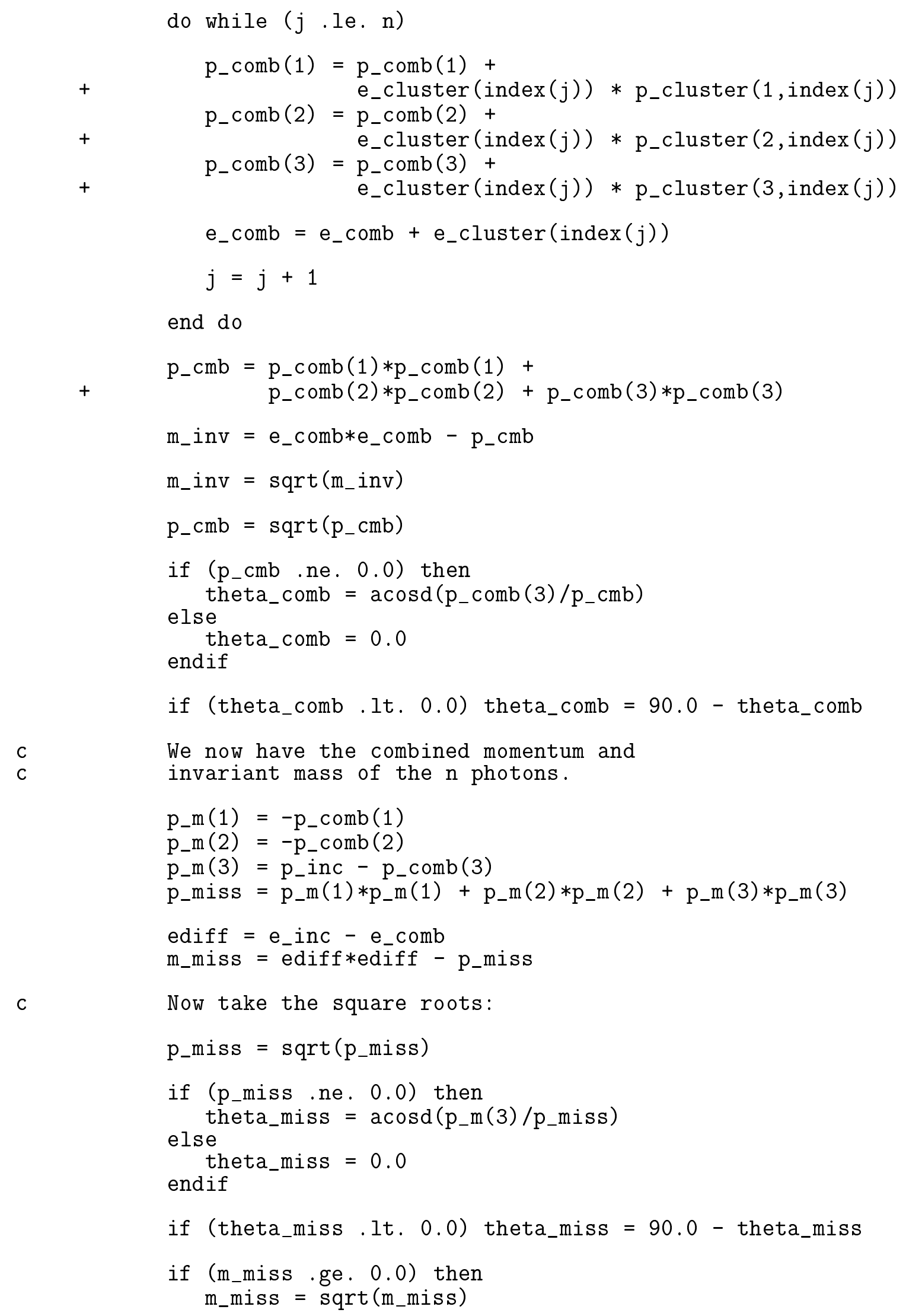




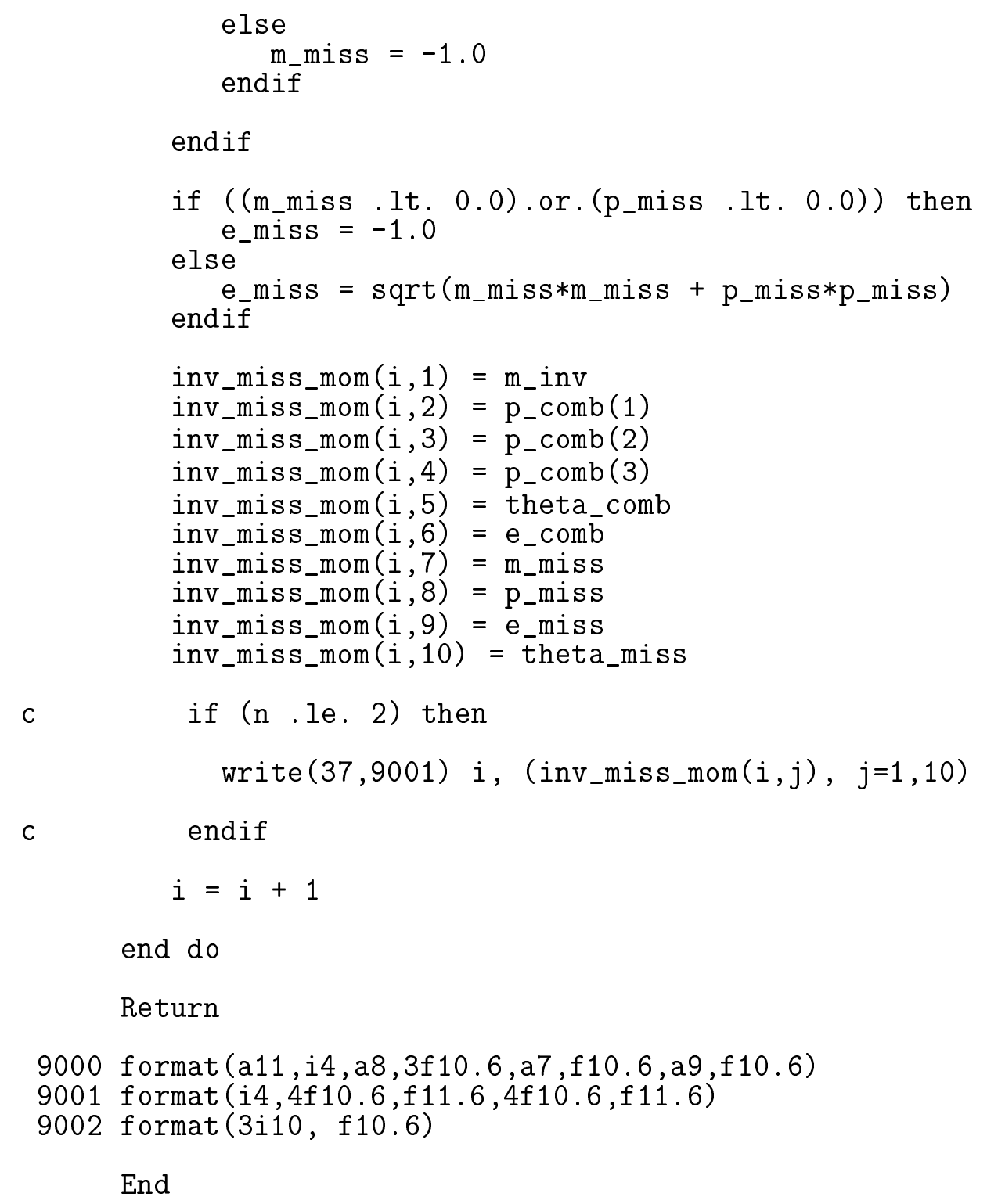

End 
The source code for the search program im_srch.f is given below. This program read information previously written to file inv_miss.dat by subroutine inv_miss_all, and picked out the combinations of clusters that looked like candidates for being various particle types. Note that im_srch was a separate FORTRAN program independent of the analyzer software. The user is queried interactively for the event number, which he/she must know beforehand. A negative value for the event number will cause the program to search the data file and determine which event numbers are present in the file. The program im_srch.f calls a subroutine called search to do the actual searching. The source code for both the main program and the subroutine are included below.

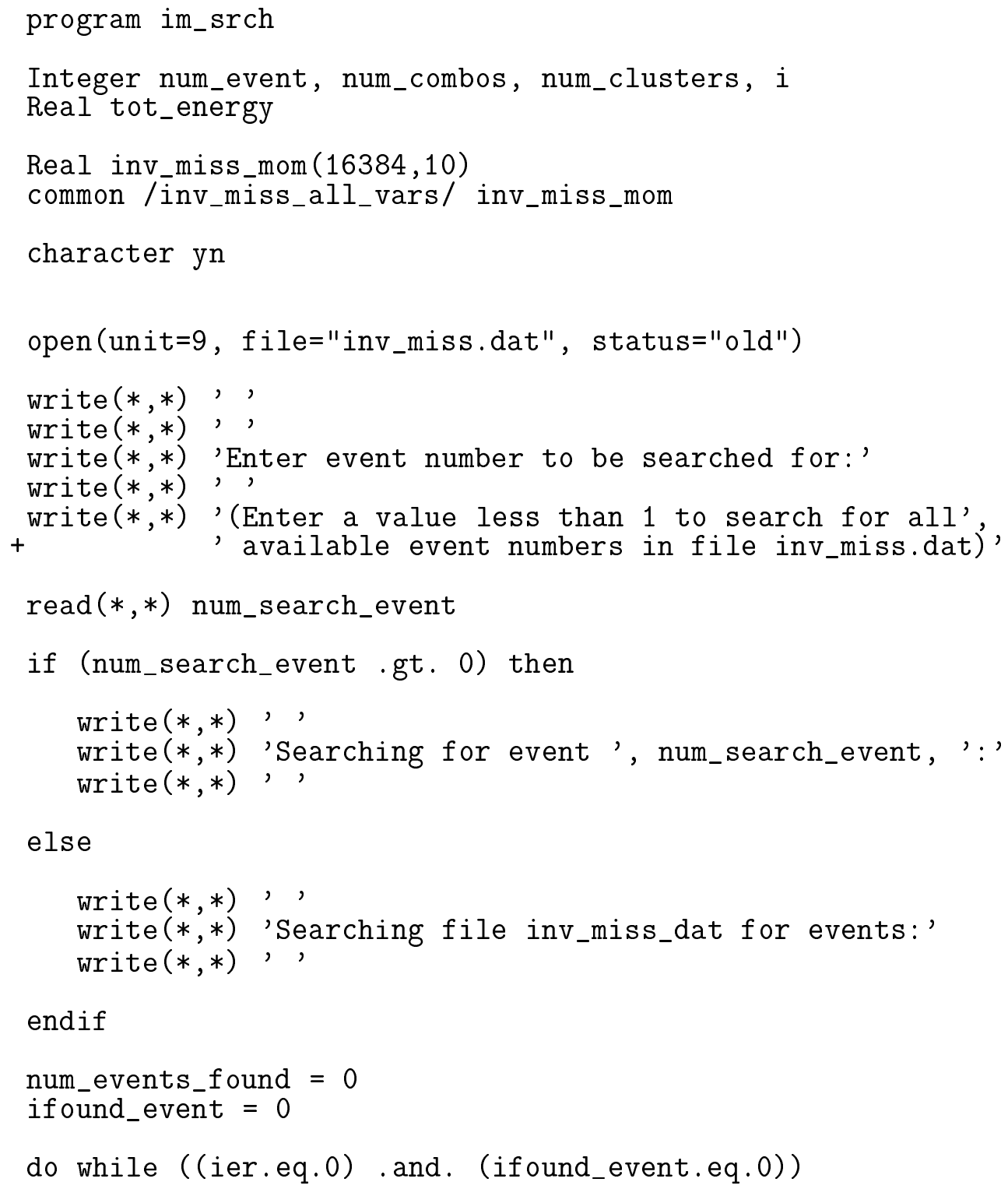




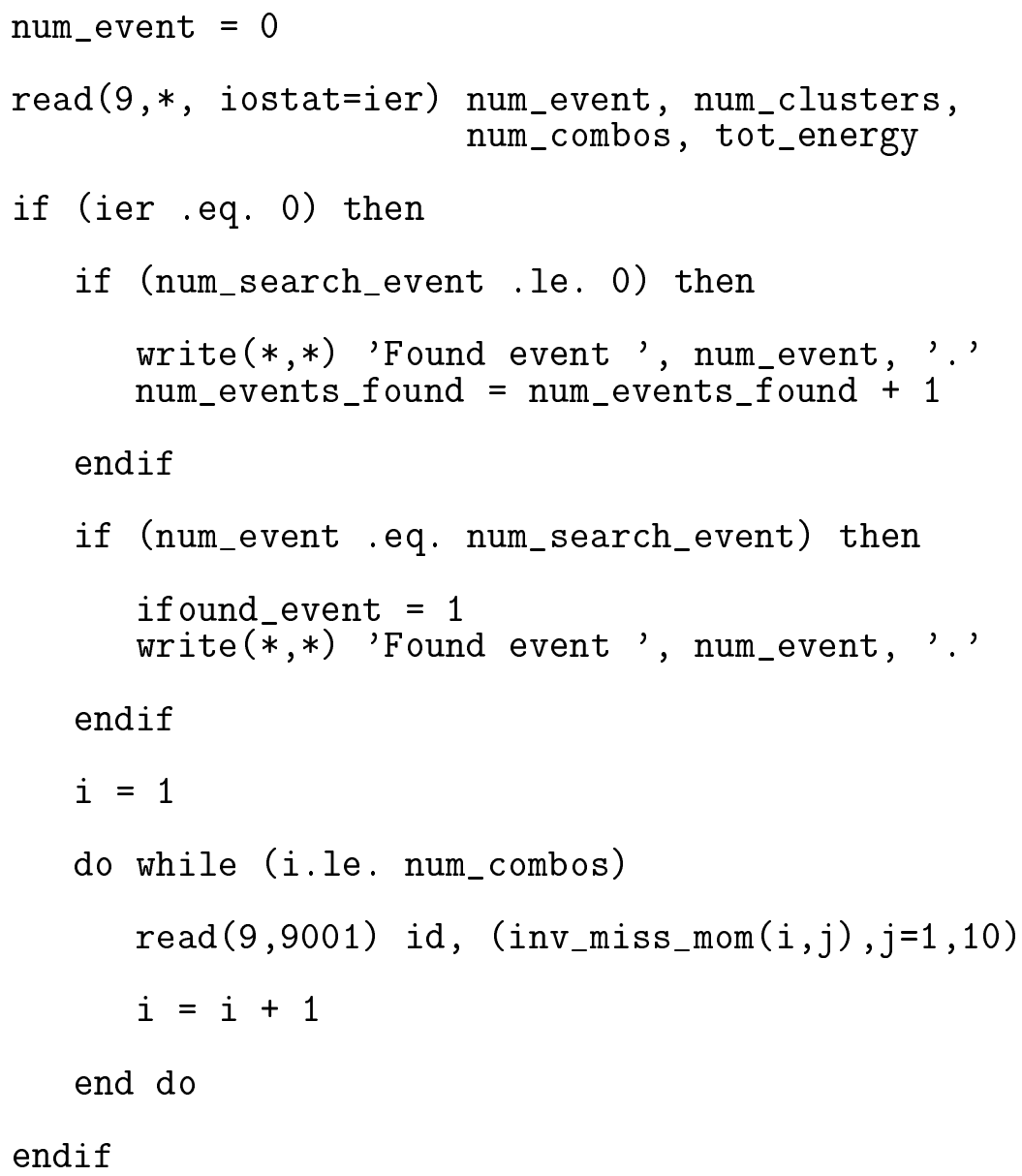




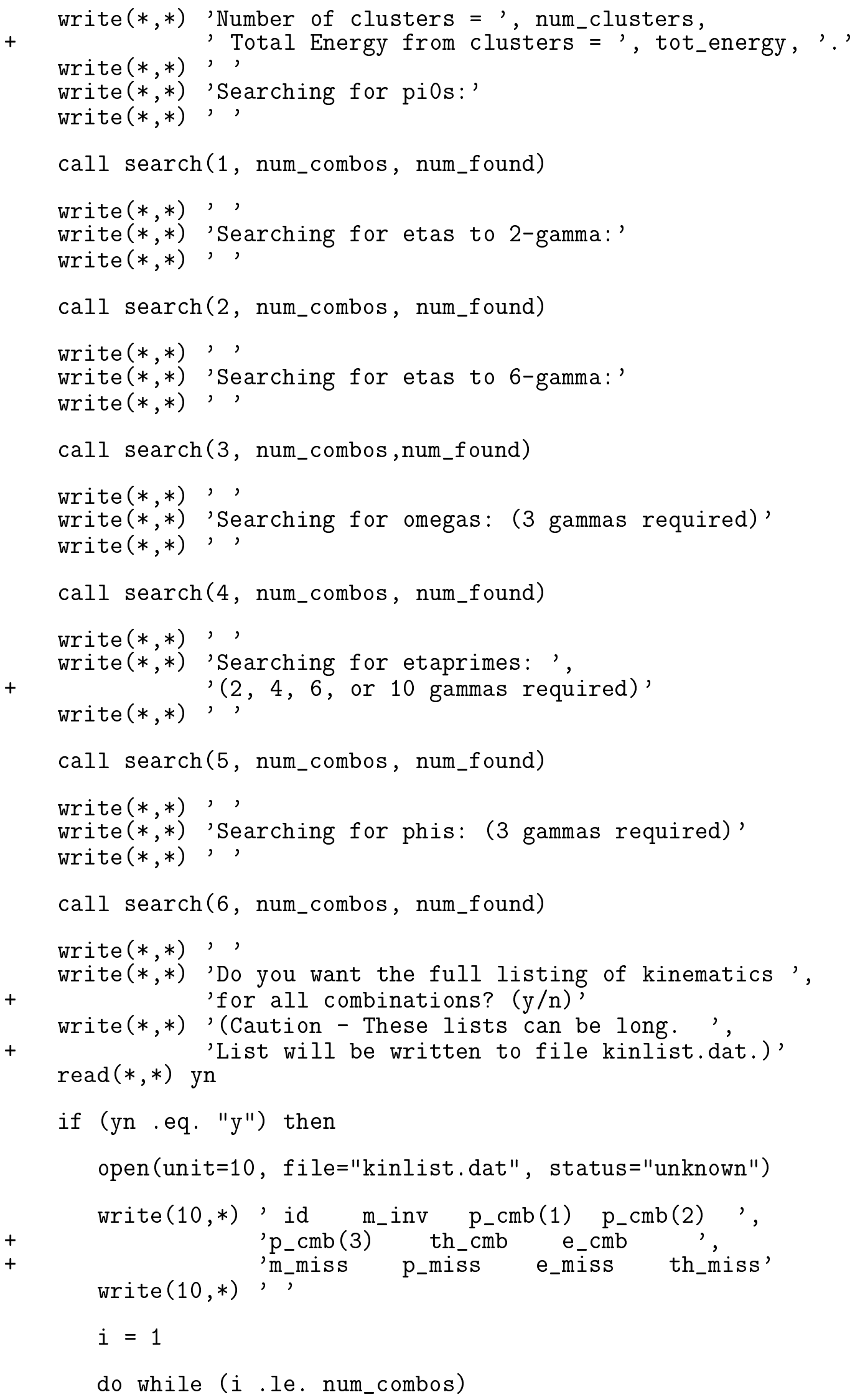




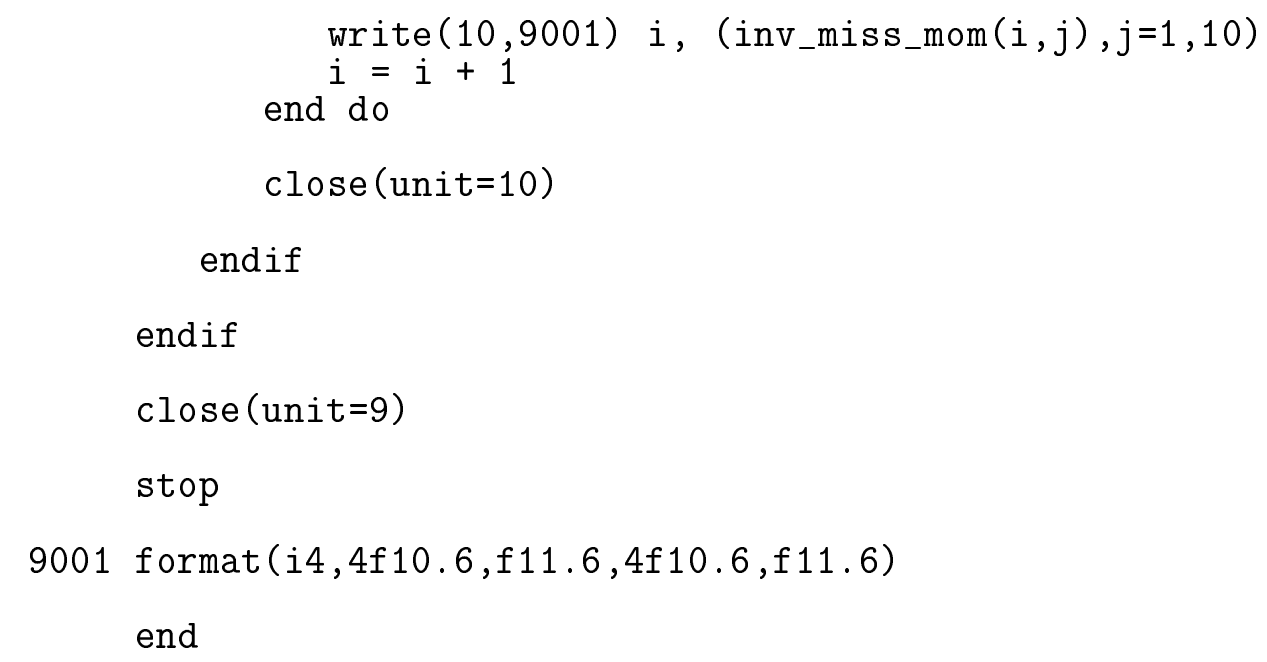




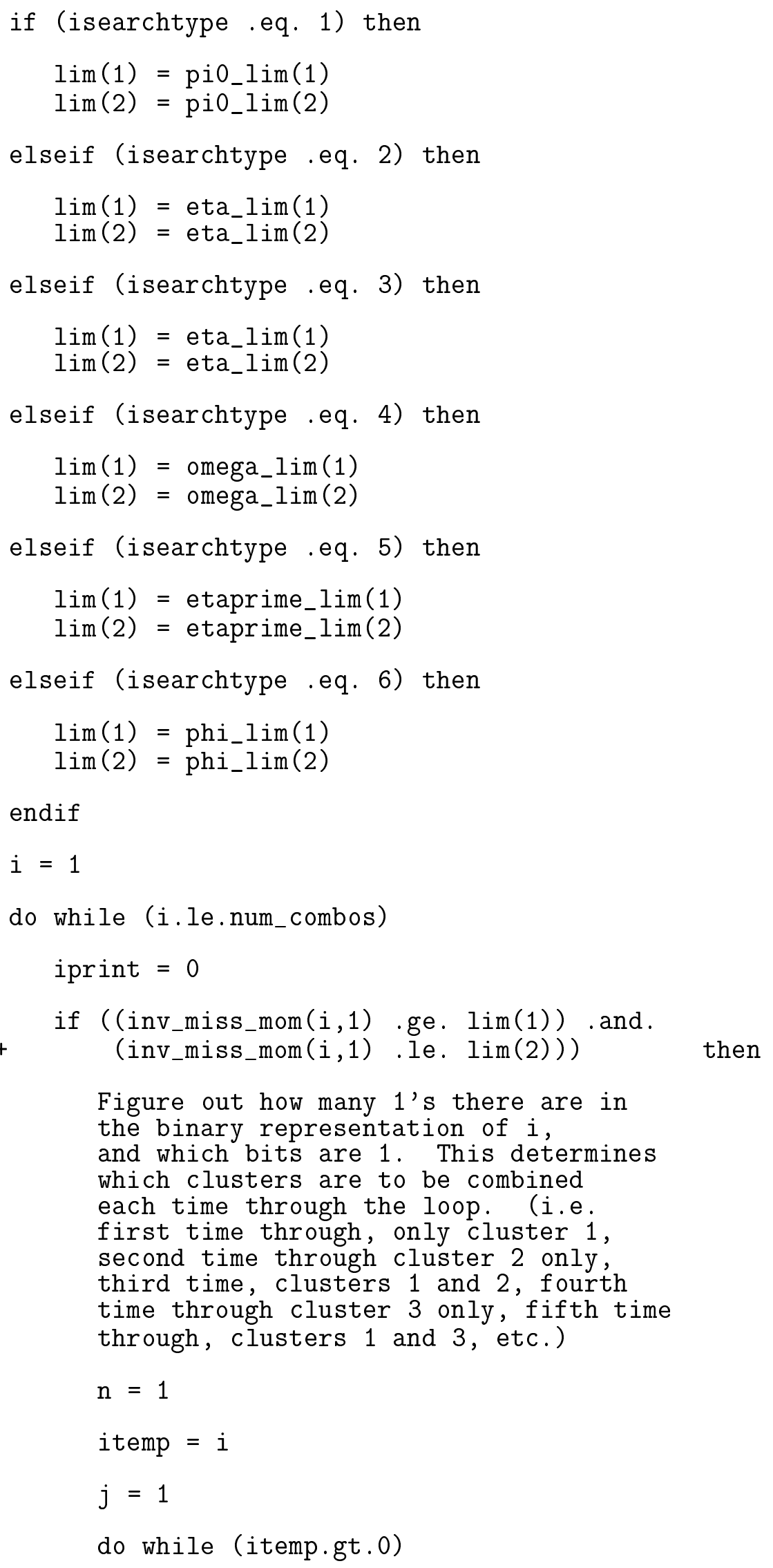




$$
\begin{aligned}
& \text { idigit }=\text { iand (itemp, 1) } \\
& \text { index }(n)=j \\
& n=n+\text { idigit } \\
& \text { itemp }=\text { ishft (itemp, }-1) \\
& j=j+1 \\
& \text { end do } \\
& n=n-1
\end{aligned}
$$

end do

Restrictions on numbers of clusters for different cases:

if (isearchtype .eq. 1) then ! (pio's)

if ( $n$.eq. 2) then

iprint $=1$

endif

elseif (isearchtype .eq. 2) then ! (eta $-\rightarrow$ 2gammas)

if ( $\mathrm{n}$.eq. 2) then

iprint $=1$

endif

elseif (isearchtype .eq. 3) then ! (eta $-\rightarrow$ 3pi0's)

if ( $n$.eq. 6) then

iprint $=1$

endif

elseif (isearchtype .eq. 4) then

C

C

C

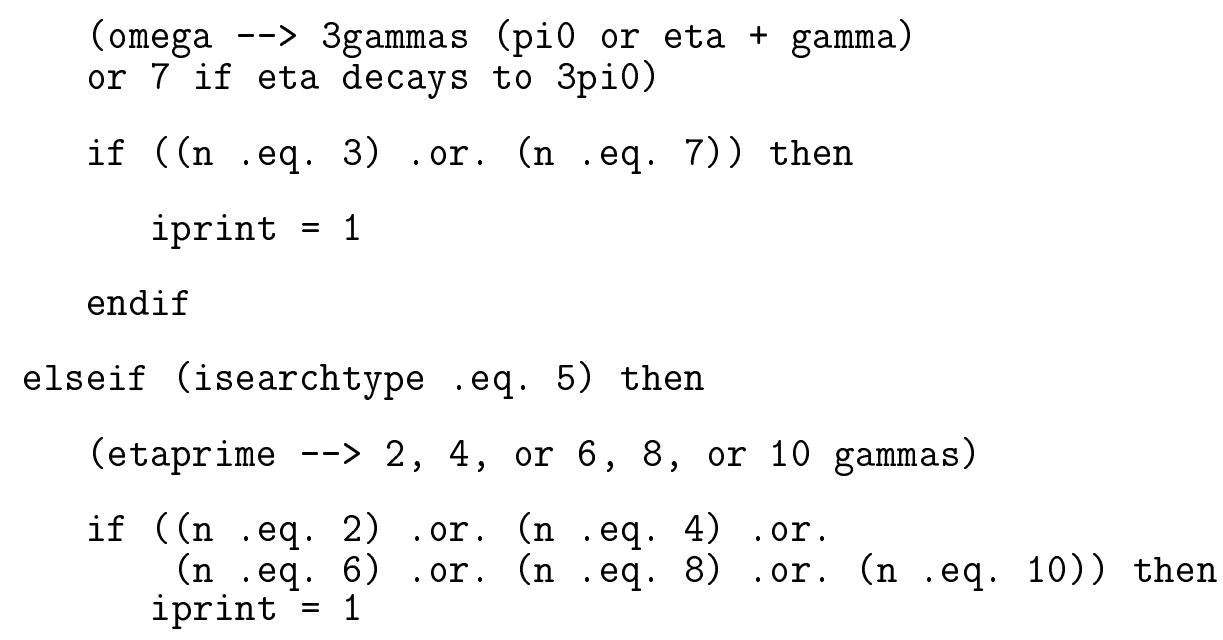




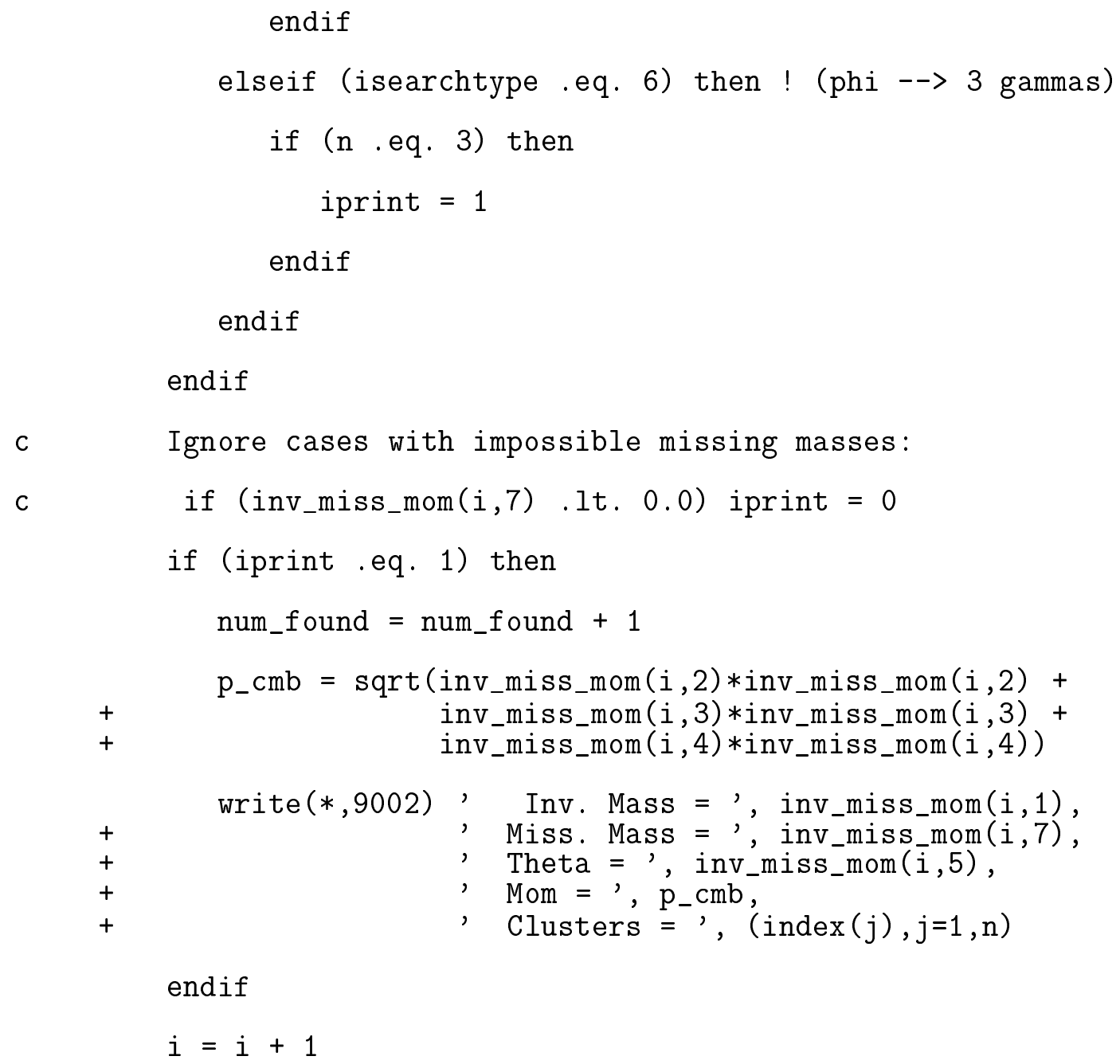

end do

if (num_found .eq. 0) then

write $(*, *)$, None Found'

9002 format (a15, f6.4, a15, f6.4, a10, f5.1, a8, f6.4, a13,14i3) end 


\section{Appendix B Pictures of Events from the SED}
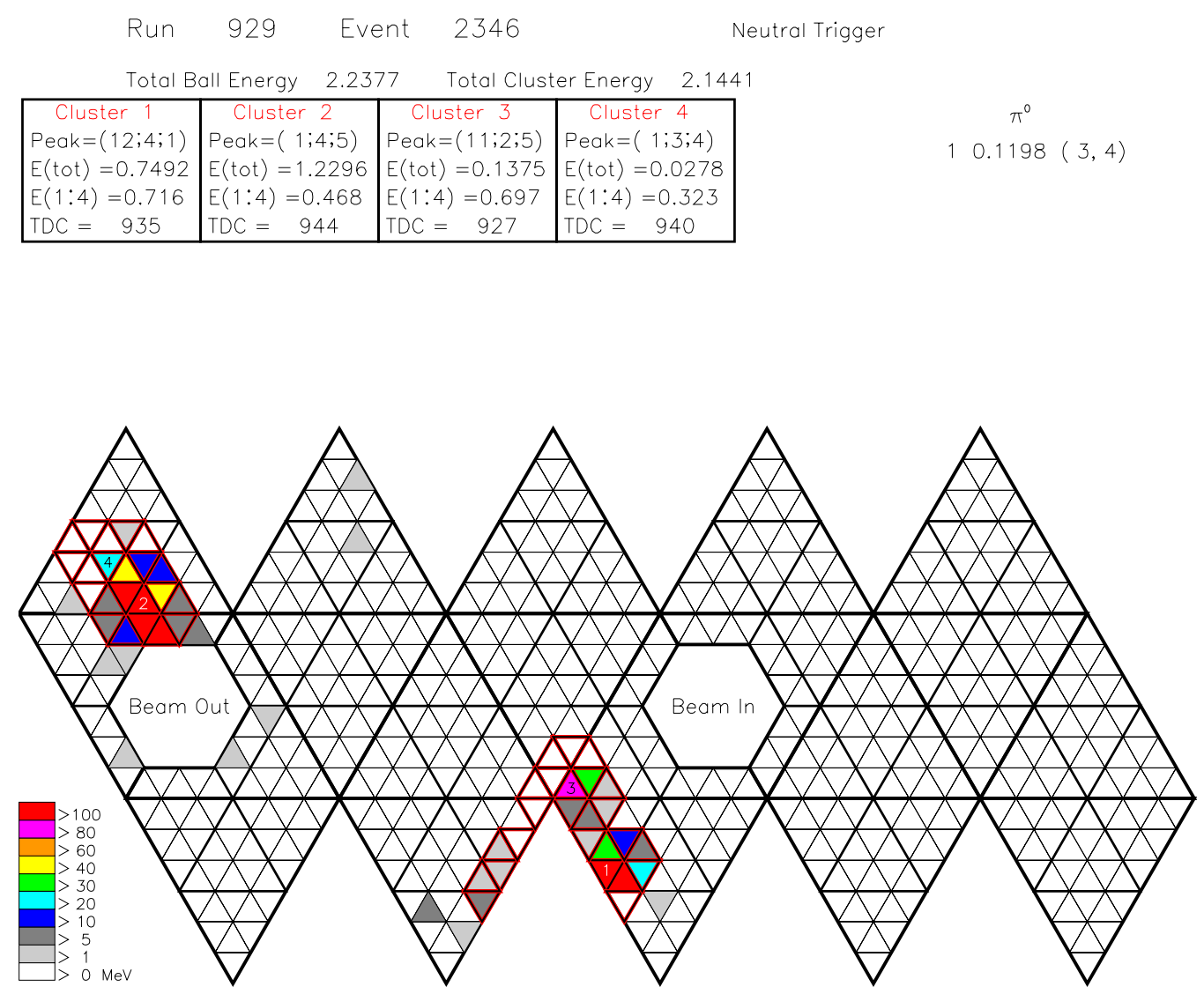

Figure 11: This is a typical $2 \pi^{\circ}$ event. There are two pairs of clusters which are back-toback in the ball. The high energies of the $\pi^{\circ}$ 's causes small opening angles and overlapping clusters. The clustering algorithm improperly handles these events most of the time, and as a result it fails to reconstruct the $\pi^{\circ}$ 's properly. This feature will become a serious problem for the Crystal Ball if it is ever used at the higher energies available in the D6 beamline. All $2 \pi^{\circ}$ events were observed to have one pion near the downstream hole. (See Fig. 5.) This is in agreement with the (as yet unpublished) $2 \pi^{\circ}$ cross section observed by the Crystal Barrel experiment at LEAR, which is strongly forward-peaked.[2] Note also the very high cluster energies which are typical of this channel. 

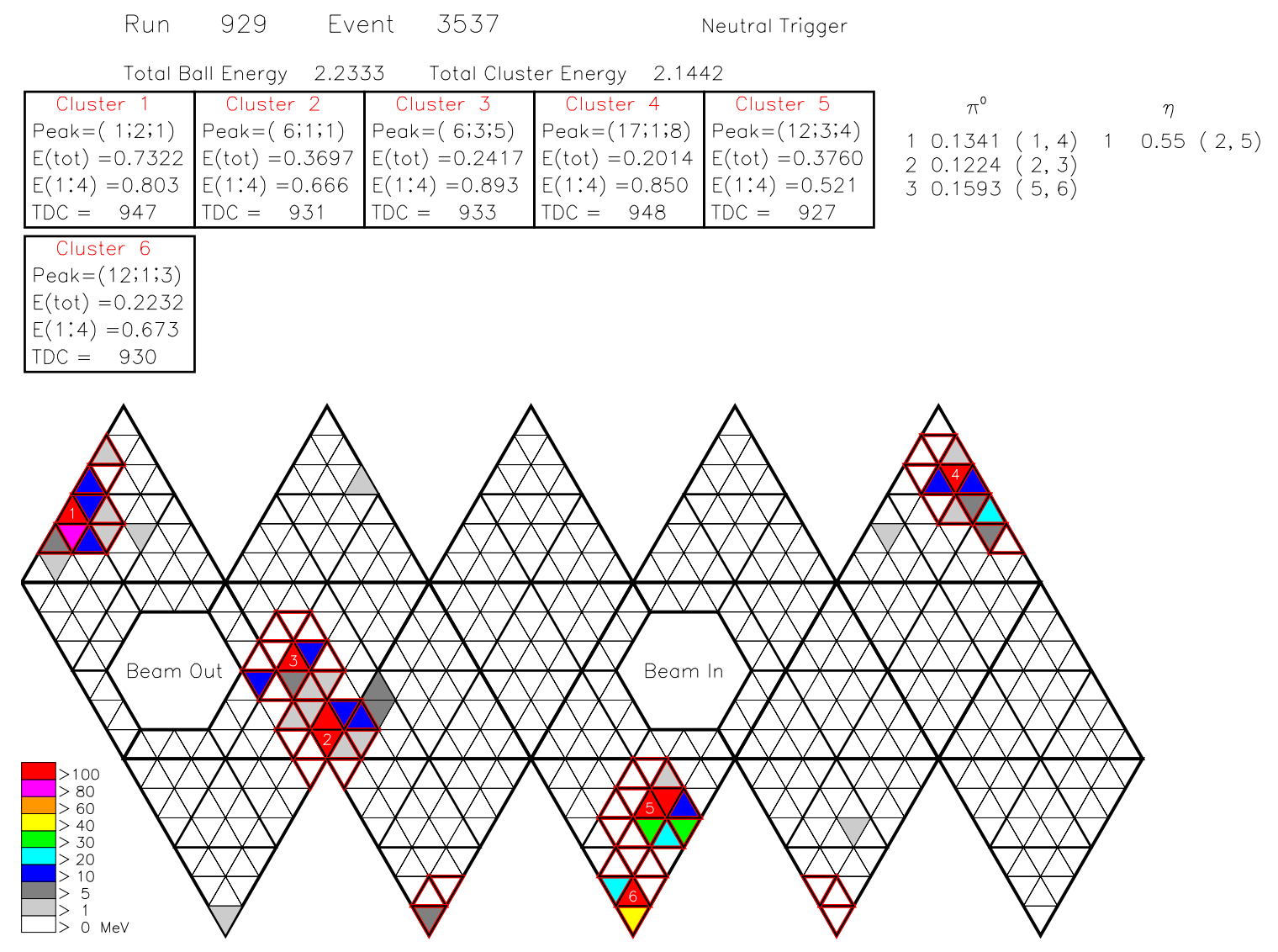

Figure 12: A typical $3 \pi^{\circ}$ event. This channel was the most common one observed. In these events the $\pi^{\circ}$ s have less energy than they do in the $2 \pi^{\circ}$ case, so opening angles are large and the clusters rarely overlap. This channel is particularly clean, and one can clearly see what is going on by looking at the SED. The pion pairs in this event are $(1,4)$, $(2,3)$, and $(5,6)$. 

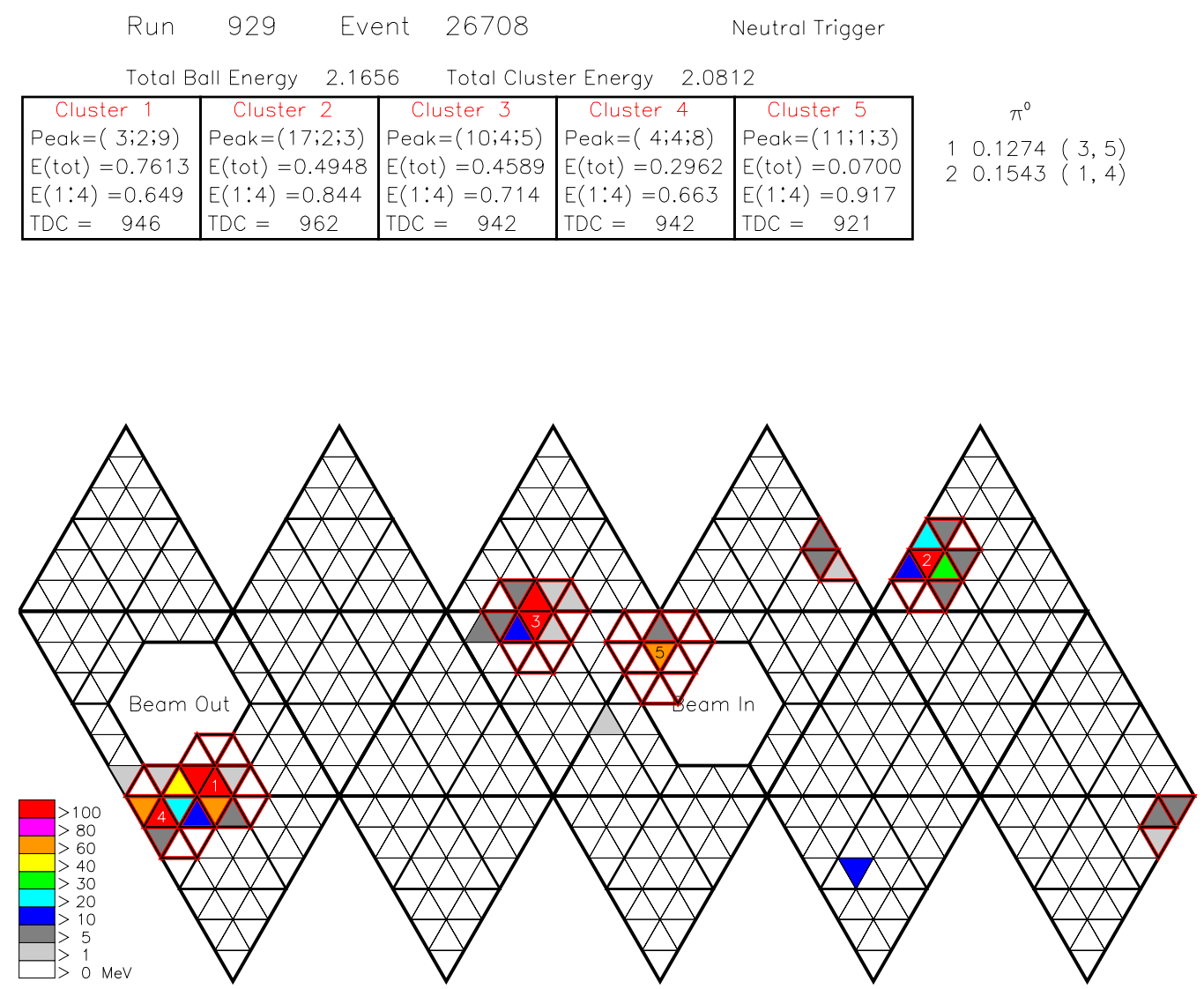

Figure 13: A simple example of an event with an odd number of clusters. In events with odd clusters, one nearly always has a phi or an omega somewhere because they decay into either $\pi^{\circ} \gamma$ or $\eta \gamma$. In this case, the reaction $\pi^{\circ}$ is clusters $(1,4)$, and the $\omega(3,5,2)$ decayed into $\pi^{\circ}(3,5)$ and $\gamma(2)$. 

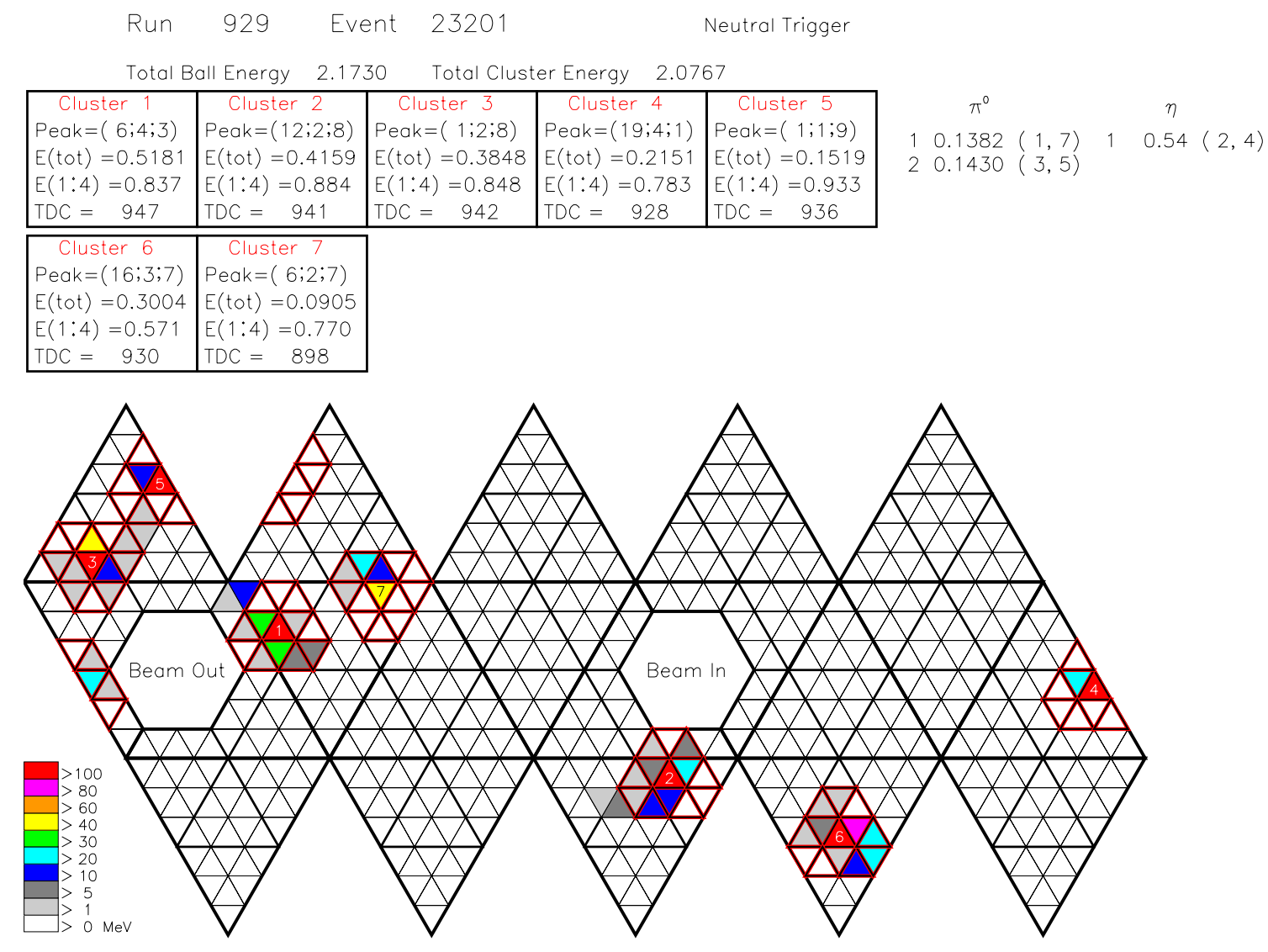

Figure 14: A more complicated odd-cluster event. This one is $\eta^{\prime} \phi$, where the $\phi(3,5,2)$ decayed into $\pi^{\circ}(3,5)$ and $\gamma(2)$, and the $\eta^{\prime}(1,7,6,4)$ decayed into $\gamma(4)$ and an $\omega(1,7,6)$ which in turn decayed into $\pi^{\circ}(1,7)$ and $\gamma(6)$. Note the enormous opening angle between the $\pi^{\circ}$ and the $\gamma$ in the $\phi$ decay. This is typical because the massive $\phi$ 's tend to come out with rather low momenta with a $550 \mathrm{MeV} / \mathrm{c}$ incident beam. As one can see, events such as these become highly non-obvious to interpret simply by eye when looking at the picture in the SED. For this event, clusters $(1,7,6,4)$ have $M_{i n v}=0.970, \theta_{l a b}=17.4^{\circ}$, $M_{\text {miss }}=0.904$ and $\theta_{\text {miss }}=86.9^{\circ}$. Clusters $(3,5,2)$ have $M_{i n v}=0.937, \theta_{\text {lab }}=90.2^{\circ}$, $M_{\text {miss }}=0.927$ and $\theta_{\text {miss }}=17.0^{\circ}$. As one can see, the determination of the angles seems to be fairly precise, but the invariant and missing masses are less reliable. (The true masses of the $\phi$ and the $\eta^{\prime}$ are $1.019 \mathrm{GeV} / c^{2}$ and $0.958 \mathrm{GeV} / c^{2}$, respectively.) 

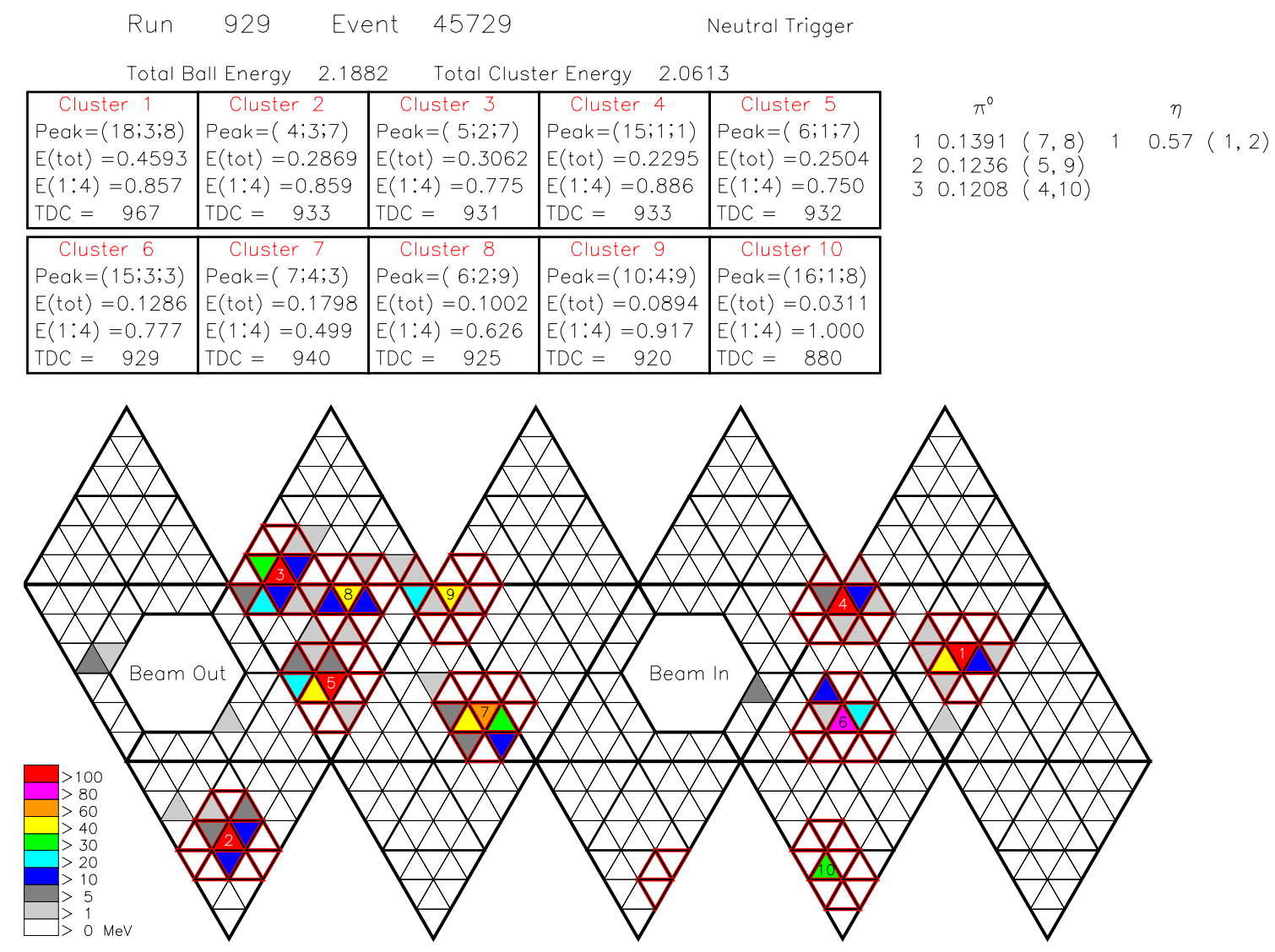

Figure 15: A 10-cluster event. The more complicated events took time and effort to disentangle, but they could be quite interesting. This one contained both of the common neutral decay modes of the $\eta$ meson in a single event. The reconstruction is $\eta \eta \pi^{\circ}$, where one $\eta$ decayed to clusters $(1,2)$, the other decayed to $\pi^{\circ}$ 's $(3,9),(5,10)$, and $(7,8)$, and the reaction $\pi^{\circ}$ is clusters $(4,6)$. The collective invariant mass of clusters $(3,9,5,10,7,8)$ is $0.581 \mathrm{GeV} / c^{2}$. When encountering events which had 10 or more clusters, there was a good chance that the event contained a $3 \pi^{\circ}$ eta decay. On the other hand, the more complex events tended to have multiple reconstruction options. This event may have instead been $\eta^{\text {prime }} 3 \pi^{\circ}$, where the three $\pi^{\circ}$ 's were $(3,9),(5,10)$, and $(4,6)$, with the $\eta^{\prime}$ having decayed via any one of $\eta^{\prime}(\omega(\eta(1,2) \gamma(8)) \gamma(7)), \eta^{\prime}(\omega(\eta(1,2) \gamma(7)) \gamma(8))$, or $\eta^{\prime}(\omega(\eta(1,7) \gamma(2)) \gamma(8))$. The $\eta \gamma$ decay mode of the $\omega$ is rare compared to the $\pi^{\circ} \gamma$ mode however. It was therefore judged that the $\eta \eta \pi^{\circ}$ option was more likely to have been the correct one. 

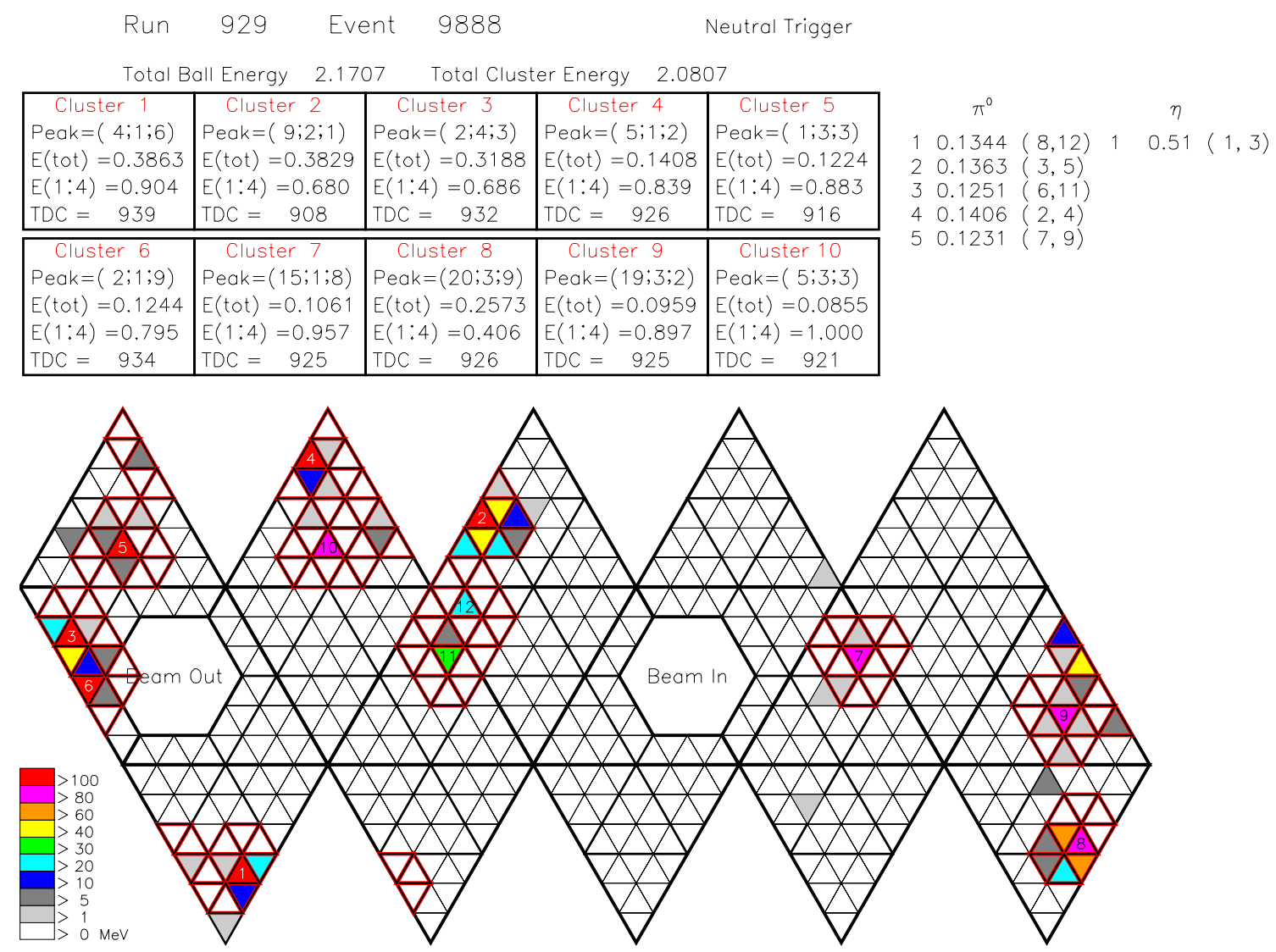

Figure 16: This is a 12-cluster event that turned out to have a unique reconstruction consisting of $\eta^{\prime} \eta$. This time the $\eta^{\prime}(1,3,6,7,8,12)$ decayed via it's favorite neutral decay mode: $\eta(1,3), \pi^{\circ}(6,7), \pi^{\circ}(8,12)$, and the $\eta(2,4,5,11,9,10)$ decayed to $\pi^{\circ}(2,4), \pi^{\circ}(5,11)$, and $\pi^{\circ}(9,10)$. The $\eta^{\prime}(1,3,6,7,8,12)$ clusters had $M_{i n v}=0.916, \theta_{l a b}=50.1^{\circ}, M_{m i s s}=0.533$ and $\theta_{\text {miss }}=86.4^{\circ}$. The $\eta(2,4,5,11,9,10)$ clusters had $M_{\text {inv }}=0.586, \theta_{\text {lab }}=75.0^{\circ}, M_{\text {miss }}=0.903$ and $\theta_{\text {miss }}=58.0^{\circ}$. The agreement between the angles is good to within about $8-11^{\circ}$, which is acceptable when so many clusters are being combined together. There were no other reconstruction options that accounted for all twelve clusters for this event. Note that the single event display was only designed to show cluster boxes for up to ten clusters in an event. The boxes for clusters 11 and 12 are therefore not included in the display above. 


\section{Appendix C Addendum: Possible $K_{l} K_{s}$ Signal}

Figure 17 shows a possible signal for the reaction $\bar{p}+p \rightarrow K_{L} K_{S}$. The plot is of the total invariant mass of all clusters for events satisfying the requirements of a neutral trigger, antiproton time of flight in S2, four clusters that reconstruct as two $\pi^{\circ}$ 's in the ball, and a total missing mass of between $300-700 \mathrm{MeV} / \mathrm{c}^{2}$. There are six events in Run 929 that are clustered near the mass of the $K_{S}$. This is the only neutral signal shown in this report for which less than $2.0 \mathrm{GeV}$ total energy was required in the ball. The $a_{4}(2040)$ should contribute to this reaction channel at this beam momentum. If so, then this may indicate a strange decay of a nonstrange meson resonance.

Figure 17: Invariant mass for 4 -cluster $2 \pi^{\circ}$ events with $K_{L}$ missing mass.

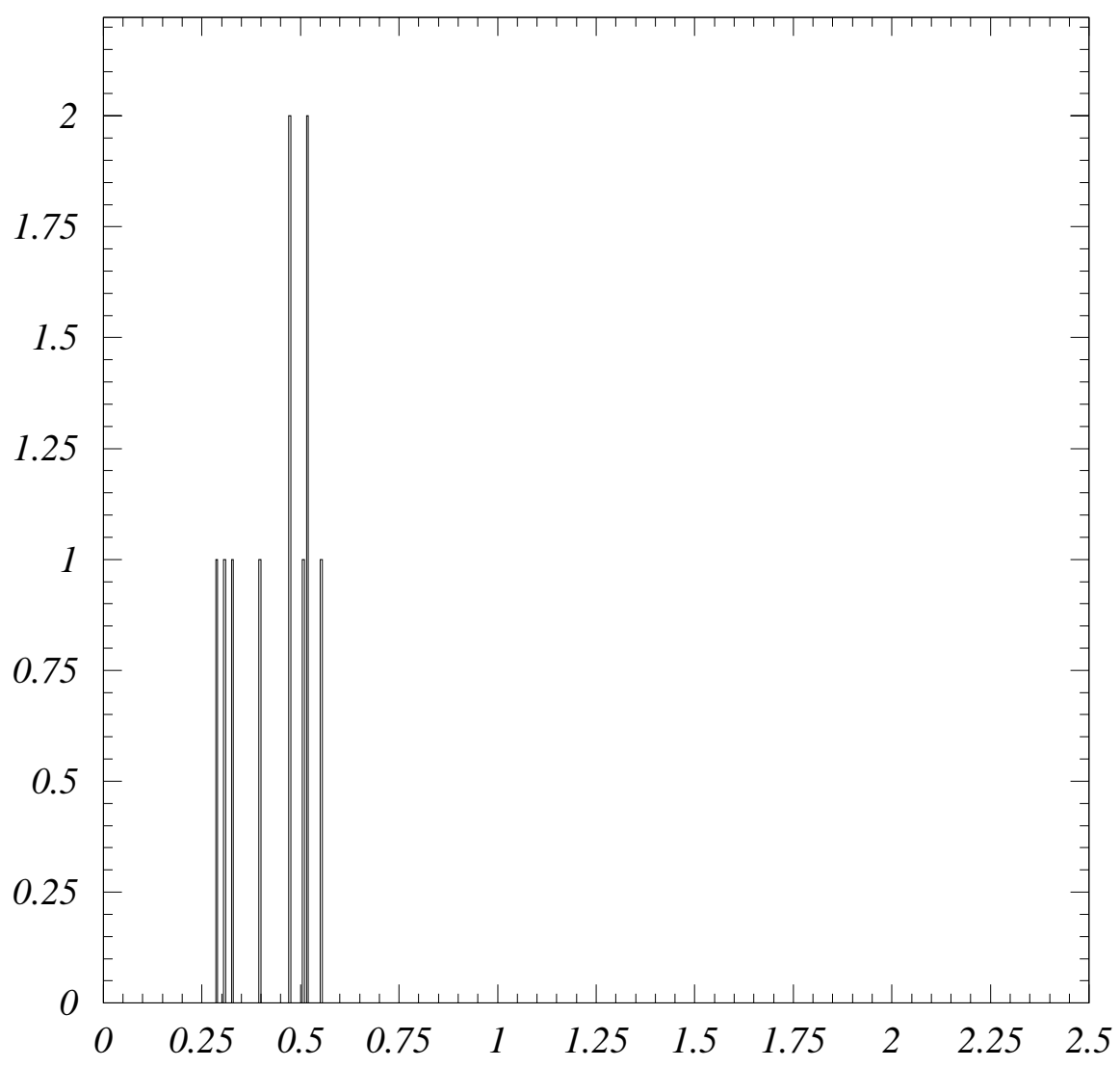

